\title{
PRESERVATION OF BOUNDED GEOMETRY UNDER SPHERICALIZATION AND FLATTENING: QUASICONVEXITY AND $\infty$-POINCARÉ INEQUALITY
}

\author{
Estibalitz Durand-Cartagena and Xining Li \\ UNED, ETSI Industriales, Departamento de Matemática Aplicada \\ Juan del Rosal 12, Ciudad Universitaria, 28040 Madrid, Spain; edurand@ind.uned.es \\ Sun Yat-sen University, Department of Mathematics \\ Guangzhou 510275, P. R. China; lixining3@mail.sysu.edu.cn
}

\begin{abstract}
In this work we explore the preservation of quasiconvexity and $\infty$-Poincaré inequality under sphericalization and flattening in the metric setting. The results developed in [22] show the preservation of Ahlfors regularity, doubling property and the $p$-Poincare inequality for $1 \leq p<\infty$ under the sphericalization and flattening transformations provided the underlying metric space is annularly quasicovex. In this work, we propose a weaker assumption to still preserve quasiconvexity and $\infty$-Poincaré inequality, called radially star-like quasiconvexity (corresponding to sphericalization) and meridian-like quasiconvexity (corresponding to flattening) extending in particular a result in [8] to a wider class of metric spaces and covering the case $p=\infty$ in [22].
\end{abstract}

\section{Introduction}

The process of obtaining the Riemann sphere from the complex plane, and vice versa, was generalized in the metric setting by using sphericalization and flattening. See the work [2] by Buckley and Balogh. These conformal transformations are dual to each other, and the performance of sphericalization followed by flattening or vice versa results in a metric space that is biLipschitz equivalent to the original space.

The advantage of considering these transformations comes partly from the fact that some results in analysis are easier to establish, either for bounded spaces or for unbounded ones; see for example [20]. Indeed, the paper [5] established equivalence between unbounded uniform domains and Gromov hyperbolic spaces, and their technique needed the uniform domain to be unbounded. The paper [20] used the sphericalization and flattening techniques to transform bounded uniform domains into unbounded uniform domains, and hence succeeded in extending the results of [5] to bounded uniform domains. As another example, recently the paper [1] proposed a notion of prime end boundary for bounded domains in the metric setting, and such a prime end boundary was the principal focus of the study of Dirichlet problems in the metric setting in [14]. However, the results in [14] needed the domain to be

https://doi.org/10.5186/aasfm.2017.4221

2010 Mathematics Subject Classification: Primary 31E05; Secondary 30L10, 30L99.

Key words: Sphericalization, flattening, doubling, Poincaré inequality, quasiconvexity, annularly quasiconvex, radially star-like quasiconvex spaces, meridian-like quasiconvex spaces.

The research of the first author is partially supported by the grant MTM2012-34341 (Spain) and 2016-MAT09 (UNED, ETSI Industriales) and the research of the second author was partially supported by the NSF grant DMS-1200915. This research began during the visit of the authors to Institut Mittag-Leffler, and continued during the visit of the first author to the Department of Mathematical Sciences at University of Cincinnati in the Spring 2014. They wish to thank these institutions for their kind hospitality. 
bounded. To establish solutions to the Dirichlet problem for unbounded domains in the metric setting, the thesis [13] used the procedure of sphericalization to transform the unbounded domain into a bounded domain, consider the prime end boundary of the resulting bounded domain, and then pulled back this prime end boundary via flattening to construct the prime end boundary of the unbounded domain, thus circumventing the problem related to non-compactness of the boundary.

A very natural problem is therefore to study which geometric properties are preserved under these transformations. In this work we will focus on the preservation of $\infty$-Poincaré inequality and quasiconvexity, a metric property of the space that ensures that one can connect two points in the space by a curve whose length is bounded by a universal constant times the distance between the two points. The geometry of $X$ at small scales is similar to the one of its sphericalized version. The differences arise at large scales. For example, local quasiconvexity is preserved under flattening and sphericalization (see [8, Proposition 4.3.]). In contrast, as shown in [8, Example 6.2.], (global) quasiconvexity is not generally preserved under these deformations. Buckley, Herron and Xie proved in [8] that, under the additional hypothesis of annular quasiconvexity, quasiconvexity and annular quasiconvexity are preserved under sphericalization and flattening.

Examples of annularly quasiconvex spaces include upper Ahlfors regular Loewner spaces (such as Carnot groups and certain Riemannian manifolds with non-negative Ricci curvature) or complete Ahlfors $Q$-regular metric measure spaces supporting a $p$-Poincaré inequality for some $p<Q$ (see [21]). However, there are some simple and natural examples of quasiconvex spaces that are not annularly quasiconvex, but whose sphericalized and flattened versions are still quasiconvex. The real line, the Euclidean infinite strip $\mathbf{R} \times[-1,1]$, and some classes of metric trees are some examples.

Motivated by these examples, we define a new class of metric spaces that encompasses annularly quasiconvex spaces and for which quasiconvexity is still preserved under sphericalization and flattening. We define radially star-like quasiconvex spaces related to the process of sphericalization (see Definition 3.1) and meridianlike quasiconvex spaces linked to the process of flattening (see Definition 4.1). We will show that these two concepts are duals: a radially star-like quasiconvex space is meridian-like quasiconvex after sphericalizing, and becomes again radially star-like quasiconvex when flattenned. This duality is shown in Lemmas 4.5 and 4.4. Some of the main results of the present work are Theorem 3.4 and Theorem 4.6 that study the preservation of quasiconvexity under sphericalization and flattening, respectively. Examples 3.7 and 4.8 illustrate the sharpness of the results.

On the other hand, metric spaces endowed with a doubling measure and supporting a Poincaré inequality are nowadays considered a standard class of spaces when developing a first order differential analysis in a metric measure space setting. See for example [3], [9], [18], [16], [17] or [24] and the references therein. Li and Shanmugalingam considered the problem of transforming not only the metric, but also the measure under sphericalization and flattening and showed in [22] that if $(X, d, \mu)$ is a complete annularly quasiconvex metric measure space with $\mu$ doubling and supporting a $p$-Poincaré inequality for some $1 \leq p<\infty$, the processes of sphericalization and flattening yield doubling metric measure spaces supporting a $p$-Poincaré inequality as well. See [22, Theorem 3.3.5, Theorem 4.3.3.].

On the other hand, it was shown in [11] and [12] that supporting an $\infty$-Poincaré inequality is equivalent to $\infty$-thick quasiconvexity and also equivalent to the fact that 
given any two points on the space and a null set $N$, there exists a quasiconvex path $\gamma$ connecting the two points so that $\mathscr{L}^{1}\left(\gamma^{-1}(\gamma \cap N)\right)=0$.

Using this geometric characterization of $\infty$-Poincaré inequality and Theorems 3.4 and 4.6, we also prove in Theorem 3.6 and Theorem 4.7 the preservation of $\infty$ Poincaré inequality under sphericalization and flattening.

Moreover, by combining Theorem 3.6 and Theorem 4.7, together with the fact that the flattening (with respect to the point $\{\infty\}$ ) of a sphericalized space is biLipschitz equivalent to the original metric space, we can deduce the following main result, extending the picture to the full range $1 \leq p \leq \infty$ in [22].

Theorem 1.1. Let $(X, d, \mu)$ be an unbounded complete metric space endowed with a doubling measure and $K$-radially star-like quasiconvex with respect to a base point $a \in X$. Then $(X, d, \mu)$ supports an $\infty$-Poincare inequality if and only if the sphericalized space $\left(\dot{X}, d_{a}, \mu_{a}\right)$ supports an $\infty$-Poincare inequality.

The techniques used in the present paper are not straightforward generalizations of a single technique. One of the key tools used is an analog of the Boman type chaining arguments as developed in [15] and [4] and used in [22]. However, these chaining results are developed to deal with integrals of functions and their gradients; we do not need to deal with functions directly because of the geometric characterization of $\infty$-Poincaré inequality. We instead modify the chaining argument to construct by hand the curves that are quasiconvex and avoid null sets almost all of the parametric time.

The preprint [10] deals with preservation of $p$-Poincaré inequalities for $1 \leq p<\infty$ under sphericalization and flattening under similar hypothesis as the ones used in the present paper.

The work is organized as follows. Section 2 recalls the basic notions needed in the paper related to metric measure spaces and to sphericalization and flattening. Section 3 focuses on the preservation of $\infty$-Poincaré inequality and quasiconvexity under sphericalization while Section 4 focuses on the preservation of those same properties under flattening.

\section{Basic concepts}

2.1. Metric measure spaces. Let $(X, d)$ be a metric space. We denote open balls centered at $x \in X$ and of radius $r>0$ by $B(x, r):=\{y \in X: d(x, y)<r\}$ and closed balls by $\bar{B}(x, r):=\{y \in X: d(x, y) \leq r\}$. For $\lambda>0, \lambda B$ denotes the ball concentric with $B$ (with respect to a predetermined center) but with radius $\lambda$ times the radius of $B$. For $0<r<R, A(a, r, R)$ denotes the annulus $A(a, r, R):=$ $\bar{B}(a, R) \backslash B(a, r)$.

By a curve in $X$ we mean a continuous map $\gamma: I \rightarrow X$, where $I \subset \mathbf{R}$ is an interval. When $I=[a, b]$ for some $a, b \in \mathbf{R}$ with $a<b$, the length $\ell_{d}(\gamma)$ of $\gamma$ with respect to the metric $d$ is defined by

$$
\ell_{d}(\gamma):=\sup \sum_{k=0}^{n-1} d\left(\gamma\left(t_{k}\right), \gamma\left(t_{k+1}\right)\right)
$$

where the supremum is taken over all partitions $a=t_{0}<t_{1}<\cdots<t_{n}=b$ of the interval $[a, b]$. A curve $\gamma$ is rectifiable if $\ell_{d}(\gamma)<\infty$. We simply write $\ell(\gamma)$ if the metric is clear from the context. The image of a curve $\gamma$ will also be denoted by $\gamma$. Given two points $x, y \in X, \gamma_{x y}$ denotes a curve connecting $x$ to $y$. Given two curves $\beta$ and $\gamma$, the concatenation of $\beta$ and $\gamma$ will be denoted by $\beta \cup \gamma$. 
A metric space $(X, d)$ is $C$-quasiconvex if there is a constant $C \geq 1$ such that every pair of points $x$ and $y$ in the space can be connected by a curve $\gamma$ such that $\ell_{d}(\gamma) \leq C d(x, y)$. Such a curve is called $C$-quasiconvex. We say that $X$ is $A$-annularly quasiconvex with respect to a base point $a \in X$ if for every $r>0$, and for each pair of points $x, y \in A(a, r / 2, r)$ there is an $A$-quasiconvex curve $\gamma_{x y}$ connecting $x$ to $y$ inside the annulus $A(a, r / A, A r)$. We say that $X$ is annularly quasiconvex if there exists $A \geq 2$ such that $X$ is $A$-annularly quasiconvex for every $a \in X$. Notice that being annularly quasiconvex with respect to some $a$ does not imply that the space is annularly quasiconvex. One can consider for example the half line $X=[0, \infty)$ endowed with the euclidean metric which is annularly quasiconvex only with respect to $a=0$. Annular quasiconvexity was introduced in [21] and has been further used for example in [8], [19] and [20].

The length function associated to a rectifiable curve $\gamma:[a, b] \rightarrow X$ is $s_{\gamma}:[a, b] \rightarrow$ $[0, \ell(\gamma)]$ given by $s_{\gamma}(t)=\ell\left(\gamma_{\mid[a, t]}\right)$. Recall that every rectifiable curve admits a unique 1-Lipschitz parametrization by the arc-length $\gamma_{s}:[0, \ell(\gamma)] \rightarrow X$ such that $\gamma=\gamma_{s} \circ s_{\gamma}$. The line integral of a Borel function $\rho: X \rightarrow[0, \infty]$ over a rectifiable path $\gamma$ is defined via the formula

$$
\int_{\gamma} \rho d s:=\int_{0}^{\ell(\gamma)}\left(\rho \circ \gamma_{s}\right)(t) d t
$$

If $\rho \equiv 1$, the previous formula gives the length of $\gamma$. Given a real-valued function $u$ in a metric space $X$, a Borel function $g: X \rightarrow[0, \infty]$ is an upper gradient of $u$ if $|u(x)-u(y)| \leq \int_{\gamma} g d s$, for each rectifiable curve $\gamma$ joining $x$ and $y$ in $X$.

Let $(X, d, \mu)$ be a metric measure space, with $\mu$ a Borel measure on $X$. The measure $\mu$ is doubling if balls have finite positive measure and there is a constant $C_{\mu} \geq 1$ such that

$$
\mu(2 B) \leq C_{\mu} \mu(B)
$$

for all balls $B$. Let $1 \leq p \leq \infty$. We say that $(X, d, \mu)$ supports a $p$-Poincaré inequality if each ball in $X$ has finite and positive measure and there are constants $C, \lambda>0$ such that for every open ball $B$ in $X$, for every measurable function $u$ on $B$, and for every upper gradient $g$ of $u$ we have

$$
\frac{1}{\mu(B)} \int_{B}\left|u-u_{B}\right| d \mu \leq C \operatorname{rad}(\lambda B)\left(\frac{1}{\mu(\lambda B)} \int_{\lambda B} g^{p} d \mu\right)^{1 / p}
$$

if $1 \leq p<\infty$, and

$$
\frac{1}{\mu(B)} \int_{B}\left|u-u_{B}\right| d \mu \leq C \operatorname{rad}(\lambda B)\|g\|_{L^{\infty}(\lambda B)},
$$

if $p=\infty$. Here, for arbitrary $A \subset X$ with $0<\mu(A)<\infty$ we write $u_{A}=\frac{1}{\mu(A)} \int_{A} u d \mu$.

Recall the following characterization of $\infty$-Poincaré inequality, which is intimately connected to quasiconvexity.

Theorem 2.1. [11, Theorem 3.1.] Suppose that $X$ is a locally complete metric space supporting a doubling Borel measure $\mu$. Then the following conditions are equivalent:

(a) $X$ supports an $\infty$-Poincaré inequality.

(b) There is a constant $C \geq 1$ such that, for every null set $N$ of $X$, and for every pair of points $x, y \in X$ there is a $C$-quasiconvex path $\gamma$ in $X$ connecting $x$ to $y$ with $\gamma \notin \Gamma_{N}^{+}$, that is, $\mathscr{L}^{1}\left(\gamma^{-1}(\gamma \cap N)\right)=0$. 
The interested reader can find in [18] a discussion of the recent advances in the field of analysis on metric measure spaces, including those in [11] and [22] (see [18, Chapter 14]).

2.2. Sphericalization and flattening. Sphericalization and flattening are conformal deformations that generalize in the context of metric spaces, the process of obtaining the Riemann sphere from the Euclidean plane and vice versa. They were introduced by Balogh and Buckley in [2] and further studied in [8] and [20].

If $X$ is an unbounded locally compact metric space, one can define its one-point compactification $\dot{X}=X \cup\{\infty\}$, where the topology on $\dot{X}$ is given by the union of the topology on $X$ and the collection of all sets that are complements in $\dot{X}$ of compact subsets of $X$ (see for instance, [23, Theorem 29.1]). Recall that a space $X$ is said to be locally compact if given $x$ in $X$, there is a neighborhood $V$ of $x$ such that $\bar{V}$ is compact.

Fixing points $a, c \in X$, one can define a spherical density $d_{a}$ on $\dot{X}$, and a flattening density $d^{c}$ on the punctured space $X^{c}=X \backslash\{c\}$. See the Table 1 for the precise formulas. As shown in [2], there exist metrics $\widehat{d}_{a}$ and $\widehat{d^{c}}$ bi-Lipschitz equivalent to the densities $d_{a}$ and $d^{c}$ respectively. Since there is no closed formula for the metrics $\widehat{d}_{a}$ and $\widehat{d}^{c}$, we will use for convenience the densities $d_{a}$ and $d^{c}$ for defining balls in $\dot{X}$ and $X^{c}$. Notice that the density function $d_{a}$ we use satisfies the criterion of sphericalizing function $g(t)=(1+t)^{-2}$ defined in $[2]$. The space $\left(\dot{X}, \widehat{d}_{a}\right)$ is a bounded metric space with $\operatorname{diam}(\dot{X})=1$, and is called the sphericalization of $(X, d)$, while $\left(X^{c}, \widehat{d}^{c}\right)$ is an unbounded metric space (if $c$ is non-isolated) called the flattening of $(X, d)$. As shown in [6], the metric space resulting from flattening with respect to the point $\{\infty\}$ the (bounded) sphericalized space $\left(\dot{X}, \widehat{d}_{a}\right)$ is bi-Lipschitz equivalent to the (unbounded) space $(X, d)$, making sphericalization and flattening dual transformations.

From a technical point of view, one of the main difficulties that one might encounter is how to transform objects that involve the critical point $\{\infty\}$. In complex analysis, we know that we can use Möbius transformations to move $\{\infty\}$ to any other point and still preserve the same geometric properties. But in a general metric setting, one has to analyze the critical point $\{\infty\}$ separately.

\begin{tabular}{|c|c|c|}
\hline $\mathrm{X}$ & $\dot{X}:=X \cup\{\infty\}$ & $a \in X$ \\
\hline$d$ & $d_{a}(x, y)= \begin{cases}\frac{d(x, y)}{[1+d(x, a)][1+d(y, a)]} & \text { if } x, y \in X, \\
\frac{1}{1+d(x, a)} & \text { if } x \in X, y=\infty, \\
0 & \text { if } x=\infty=y .\end{cases}$ & $d^{c}(x, y)=\frac{d(x, y)}{d(x, c) d(y, c)} \quad$ if $x, y \in X^{c}$ \\
\hline$B(x, r)$ & $\ell_{d_{a}}(\gamma)=\int_{0}^{\ell(\gamma)} \frac{1}{\left[1+d\left(\gamma_{s}(t), a\right)\right]^{2}} d s(t)$ & $\ell_{d^{c}}(\gamma)=\int_{0}^{\ell(\gamma)} \frac{1}{d\left(\gamma_{s}(t), c\right)^{2}} d s(t)$ \\
\hline$\ell(\gamma)$ & $\mu_{a}(A)=\int_{A \backslash\{\infty\}} \frac{1}{\mu(B(a, 1+d(z, a)))^{2}} d \mu(z)$ & $\mu^{c}(A)=\int_{A} \frac{1}{\mu(B(c, d(c, z)))^{2}} d \mu(z)$ \\
\hline$\mu$
\end{tabular}

Table 1. Relevant formulas for flattening and sphericalization.

In the sequel, it will be also useful to know how a curve and its corresponding length change under the sphericalization and flattening processes. Let $\gamma$ be a rectifiable curve in a rectifiably connected unbounded metric space $X$. Under sphericalization $\gamma$ corresponds to $\dot{\gamma}:[0, \ell(\gamma)] \rightarrow \dot{X}$ defined by $\dot{\gamma}(t)=\gamma_{s}(t)$, where $\gamma_{s}$ is the arc-length parametrization of $\gamma$ with respect to the original metric $d$. By an abuse of notation we will denote the corresponding curve in $\dot{X}$ by $\gamma$ as well. One 
can check (see [2, Proposition 2.6]) that $\gamma$ is rectifiable with respect to the metric $\widehat{d}_{a}$ if it is rectifiable with respect to the original metric $d$. The formulas for the length of $\dot{\gamma}$ with respect to the metric $\widehat{d}_{a}$ and the length in the metric $\widehat{d}^{c}$ of a given a curve $\gamma:[a, b] \rightarrow X^{c}$ (notice that $X^{c} \subset X$ ) are given in the Table 1.

If $X$ is equipped with a Borel-regular measure $\mu$ such that measures of non-empty open sets are positive and measures of bounded sets are finite, we can construct an induced measure $\mu_{a}$ on $\dot{X}$ and $\mu^{c}$ on $X^{c}$. It was shown in [22, Proposition 3.6] that if $\mu$ is doubling, then so are $\mu_{a}$ and $\mu^{c}$, from which follows in particular that because $\dot{X}$ is bounded, $\mu_{a}(\dot{X})$ is finite. All the relevant formulas are gathered in Table 1. In what follows, for further clarification, the base point in the sphericalization will be denoted by $a \in X$, and the one in the flattening by $c \in X$.

Unless otherwise stated, the letter $C$ denotes various positive constants whose exact values are not important for the purposes of this paper, and its value might change even within a line.

\section{Preservation of quasiconvexity and $\infty$-Poincaré inequality under sphericalization: radially star-like quasiconvex spaces}

In [8, Section 6] Buckley, Herron and Xie studied the preservation of quasiconvexity (and annular quasiconvexity) under sphericalization under the hypothesis of annular quasiconvexity. In view of the fact that natural examples such as the real line or a broad class of metric trees are not annularly quasiconvex, our aim in this section is to introduce a larger class of metric spaces that encompasses such examples and whose transformations still preserve properties such as quasiconvexity or $\infty$-Poincaré inequality.

To motivate our definition, we go back to star-like domains that appear naturally in complex analysis as sets for which the line segment connecting any point to a fixed base point lies entirely inside the domain.

A relaxation of the star-likeness property, rough star-likeness, is enjoyed by some proper geodesic Gromov hyperbolic spaces. A metric space is $K$-roughly star-like with respect to a base point $a \in X$, if for every point $x \in X$ there exists some geodesic ray (isometric image in $X$ to $[0, \infty)$ ) emanating from $a$ whose distance to $x$ is at most $K$. This property was first named in [5], although roughly star-like spaces were called visual in [7]. Bonk, Heinonen and Koskela in [5] provided a way to transport questions in Gromov hyperbolic spaces to questions in bounded uniform spaces, and vice versa. In particular, the authors proved that if a metric space $(X, d)$ is a uniform space, the associated quasi hyperbolic metric is a proper geodesic Gromov hyperbolic space that is roughly star-like. On the other hand, if one begins with a (typically unbounded) proper geodesic roughly star-like Gromov hyperbolic space $\left(X, d_{H}\right)$ one can obtain a bounded uniform domain $\left(X, d_{\varepsilon}\right)$ depending on a sufficiently small parameter $\varepsilon>0$. In some sense, the process of flattening-sphericalization pursues a similar goal, though the uniformization metric $d_{\epsilon}$ of [5] is not the same as the metrics studied in this paper.

The following definition is inspired by roughly star-like spaces, additionally requiring that the point $x$ is connected to the geodesic ray by a controlled quasiconvex curve. Recall here that given two points $x, y \in X, \gamma_{x y}$ denotes a curve connecting $x$ to $y$.

Definition 3.1. A space is $K$-radially star-like quasiconvex with respect to a base point $a \in X$, if there exist a constant $K \geq 2$ and a radius $r_{0}>0$ such that for every $r>r_{0}$ and $x \in A(a, r / 2, r)$, there exist a base-point quasiconvex ray $\gamma_{a \infty}$, a 
point $y \in \gamma_{a \infty}$ and a quasiconvex curve $\gamma_{x y} \subset A(a, r / K, K r)$ connecting $x$ to $y$ such that

$$
\ell\left(\gamma_{x y}\right) \leq K d(a, y)
$$

Here we say that a ray $\gamma:[0, \infty) \rightarrow X$ with $\gamma(0)=a$ is base-point quasiconvex if for each $z \in \gamma, \ell\left(\gamma_{a z}\right) \leq K d(a, z)$, where $\gamma_{a z}$ is the subcurve of $\gamma$ ending at $z$.

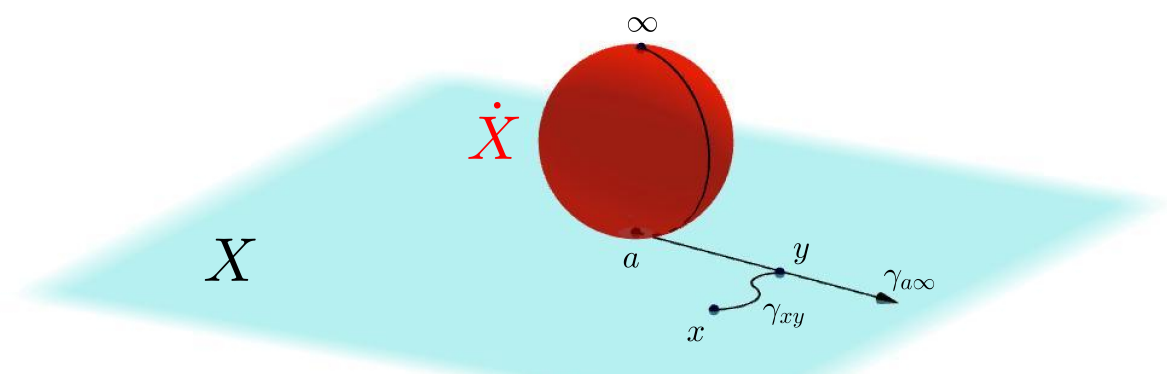

Figure 1. Radially star-like quasiconvexity.

Remark 3.2. We will always assume in this paper that our curves are parametrized by arc-length. Therefore, for a curve $\gamma:[0, \infty) \rightarrow X$ we have that $\ell\left(\gamma_{\mid[0, s]}\right)=s$.

The following lemma shows that, in a general class of metric spaces, annular quasiconvexity is stronger than radially star-like quasiconvexity. As mentioned in the Introduction, $\mathbf{R}$ is $K$-radially star-like quasiconvex but is not annularly quasiconvex.

Lemma 3.3. Let $(X, d)$ be an unbounded connected complete locally compact metric space which is annularly quasiconvex with respect to a point $a \in X$. Then $(X, d)$ is radially star-like quasiconvex with respect to $a$.

Proof. First observe that a connected annularly quasiconvex space with respect to a point $a \in X$ is quasiconvex. Indeed, let $x \neq y$ and assume $d(x, a) \geq d(y, a)$. Let us denote $B_{i}=B\left(a, 2^{-i} r\right)$ for $i \in \mathbf{N}$, where $r=d(x, a)$. Because $X$ is connected, for each $i \in \mathbf{N}$ for which $X \backslash B_{i}$ is non-empty, there exists $y_{i} \in X$ such that $d\left(a, y_{i}\right)=2^{-i} r$. Set $y_{0}=x$. By $A$-annular quasiconvexity with respect to the point $a \in X$, for each such $i \in \mathbf{N}$, there exists an $A$-quasiconvex curve $\gamma_{i} \subset X$ connecting $y_{i-1}$ to $y_{i}$ such that $\gamma_{i} \subset A\left(a, \frac{2^{-i} r}{A}, A 2^{-i} r\right)$. If $y=a$, then we choose $\gamma$ to be the concatenation of the curves $\gamma_{1}, \gamma_{2}, \cdots$, and set $\beta$ to be the constant curve starting and ending at $a$. If $y \neq a$, then let $N$ be the positive integer such that

$$
2^{-N-1} r \leq d(y, a)<2^{-N} r
$$

and let $\gamma=\gamma_{1} \cup \gamma_{2} \cup \cdots \cup \gamma_{N} \cup \beta$ be the concatenation of $\gamma_{1}, \gamma_{2}, \ldots, \gamma_{N}, \beta$, where $\beta$ is an $A$-quasiconvex curve connecting $y_{N}$ to $y$. Then the curve $\gamma$ connects $x$ to $y$ and

$$
\begin{aligned}
\ell(\gamma) & \leq \sum_{i=1}^{N} \ell\left(\gamma_{i}\right)+\ell(\beta) \leq A \sum_{i=1}^{N} d\left(y_{i-1}, y_{i}\right)+A d\left(y_{N}, y\right) \\
& \leq A \sum_{i=1}^{N+1}\left(2^{-i+1} r+2^{-i} r\right)=3 A r \sum_{i=1}^{N+1} 2^{-i}=3 A r\left(1-2^{-N-1}\right) \leq 3 A d(x, y) .
\end{aligned}
$$

In the above, if $y=a$, then $N=\infty$ and $r \approx d(x, a)=d(x, y)$. Thus we have that $X$ is quasiconvex with quasiconvexity constant $3 A$. 
We now show that $X$ is radially star-like quasiconvex. To do so, fix a point $z \in A(a, r / 2, r)$ for some $r>0$. Let $\left\{x_{n}\right\}_{n}$ be a sequence of points converging to $\infty$ with $x_{1}=z$, that is, $\lim _{n \rightarrow \infty} d\left(x_{n}, a\right)=\infty$. For each $n \in \mathbf{N}$, by the use of Arzelá-Ascoli theorem, we can find a quasiconvex curve $\gamma_{n}$ connecting $a$ to $x_{n}$ so that

$$
\ell\left(\gamma_{n}\right)=\inf _{\gamma \text { connects } a \text { to } x_{n}} \ell(\gamma) .
$$

With respect to the use of Arzelá-Ascoli theorem, we relax the requirement that curves $\gamma$ be arc-length parametrized with the requirement that $\gamma:[0, \infty) \rightarrow X$ with the restriction of $\gamma$ to $[0, \ell(\gamma)]$ is arc-length parametrized and $\gamma$ is constant on the interval $[\ell(\gamma), \infty)$. Observe that a curve constructed in this way is base-point quasiconvex, that is, for any $y \in \gamma_{a x_{n}}, \ell\left(\gamma_{a y}\right) \leq C d(a, y)$, where $\gamma_{a y}$ is the subcurve of $\gamma_{a x_{n}}$ ending at $y$. Since for every finite closed interval $I \subset[0, \infty)$, the subcurves $\gamma_{n \mid I}$ have uniformly bounded length for any $n \in \mathbf{N}$, we can extract, by Arzelá-Ascoli theorem, a subsequence $\left\{\gamma_{n_{k}}\right\}$ converging to a ray $\gamma_{a \infty}$. Observe that $\gamma_{a \infty}$ is base-point quasiconvex. To finish, for each $x \in A(a, r / 2, r)$ choose $y \in \gamma_{a \infty} \cap A(a, r / 2, r)$ and construct a quasiconvex curve $\gamma_{x y}$ in the annulus $A(a, r / A, A r)$ connecting $x$ to $y$ using the annular quasiconvexity.

In fact, the proof has shown that annular quasiconvexity implies radially star-like quasiconvexity, with radially star-like quasiconvexity constants independent of the choice of $r_{0}>0$ one would choose in the definition of radially star-like quasiconvexity.

We now state the main result of this section, namely, that quasiconvexity is preserved under sphericalization for radially star-like quasiconvex spaces.

Theorem 3.4. Let $(X, d)$ be an unbounded complete quasiconvex metric space. Let $a \in X$ be a base point on $X$, and assume $(X, d)$ is $K$-radially star-like quasiconvex with respect to a for some $K \geq 2$. Then $\left(\dot{X}, d_{a}\right)$ is quasiconvex.

Proof. Given $x_{1}, x_{2} \in \dot{X}$, we have to prove that there exists a curve $\gamma$ connecting $x_{1}$ and $x_{2}$ such that $\ell_{d_{a}}(\gamma) \leq C d_{a}\left(x_{1}, x_{2}\right)$. Notice that in the case when $d\left(x_{1}, a\right) \leq 2 r_{0}$ and $d\left(x_{2}, a\right) \leq 2 r_{0}$ with $r_{0}$ as in Definition 3.1, the original and the sphericalized metric are bi-Lipschitz on the ball $B\left(a, 2 C_{q} r_{0}\right)$ where $C_{q}$ is the quasiconvexity constant of $X$, and so we can take a quasiconvex curve $\gamma_{x_{1} x_{2}}$ with respect to the metric $d$ connecting $x_{1}$ and $x_{2}$, which is also quasiconvex with respect to the metric $d_{a}$. We now break the remaining parts of the proof into four different cases: points that are in the same annulus and far away from each other (Case 1), points that are in the same annulus and close to each other (Case 2), points that lie in different annuli (Case 3) and finally when one of the points is the point at $\infty$ (Case 4 ). The annuli that appear in the proof are considered with respect to the original metric $d$.

Fix $c^{\prime}>0$ such that $0<c^{\prime}<1 /\left(4 C_{q}\right)$ and let $x_{1}, x_{2} \in \dot{X}$. As pointed out before, we can assume that at least one of $x_{1}, x_{2}$ is not in the ball $B\left(a, r_{0}\right)$.

Before addressing the above four cases, we give the following preliminary calculations. If $\gamma$ is a quasiconvex curve in $X$ connecting two points $x_{1}$ and $x_{2}$ and lying in the annulus $A(a, r / K, K r)$ for some $r>0$, then whenever $w \in \gamma$, we have $d(w, a) \geq \frac{r}{K} \geq \frac{d\left(x_{1}, a\right)}{K^{2}}$, and thus it follows that

$$
\begin{aligned}
\ell_{d_{a}}(\gamma) & =\int_{\gamma} \frac{d s}{[1+d(\gamma(s), a)]^{2}} \leq \frac{\ell(\gamma)}{\left[1+\left(d\left(x_{1}, a\right) / K^{2}\right)\right]^{2}} \\
& \leq \frac{K^{2} r}{\left[1+d\left(x_{1}, a\right) / K^{2}\right]^{2}} \leq K^{4} d_{a}\left(x_{1}, \infty\right) .
\end{aligned}
$$


Case 1. $x_{1}$ and $x_{2}$ are in the same annulus $A(a, r / 2, r)$ for some $r>r_{0}$, with $d\left(x_{1}, x_{2}\right) \geq c^{\prime} r$. Note that by this assumption, neither of $x_{1}, x_{2}$ is the point $\infty$, and by the discussion above, $r>2 r_{0}$. By the $K$-radially star-like quasiconvex property, there exist base-point quasiconvex rays $\gamma_{a \infty}^{1}, \gamma_{a \infty}^{2}$, points $y_{1} \in \gamma_{a \infty}^{1}, y_{2} \in \gamma_{a \infty}^{2}$ and quasiconvex curves $\gamma_{x_{1} y_{1}}, \gamma_{x_{2} y_{2}}$ such that

$$
\ell\left(\gamma_{x_{1} y_{1}}\right) \leq K d\left(a, y_{1}\right) \quad \text { and } \quad \ell\left(\gamma_{x_{2} y_{2}}\right) \leq K d\left(a, y_{2}\right)
$$

Let us show that the concatenation $\gamma=\gamma_{x_{1} y_{1}} \cup \gamma_{y_{1} \infty}^{1} \cup\{\infty\} \cup \gamma_{\infty y_{2}}^{2} \cup \gamma_{y_{2} x_{2}}$ that connects $x_{1}$ and $x_{2}$ satisfies that $\ell_{d_{a}}(\gamma) \leq C d_{a}\left(x_{1}, x_{2}\right)$. Here, $\gamma_{y_{1} \infty}^{1}$ is a subcurve of $\gamma_{a \infty}^{1}$ with end points $y_{1}$ and $\infty$, and $\gamma_{y_{2} \infty}^{2}$ is the analogous subcurve for $y_{2}$. By (3.1) above, we have

$$
\ell_{d_{a}}\left(\gamma_{x_{1} y_{1}}\right) \leq K^{4} d_{a}\left(x_{1}, \infty\right)
$$

On the other hand, since $d\left(x_{1}, x_{2}\right) \geq c^{\prime} r \geq c^{\prime} d\left(x_{2}, a\right)$, and $d\left(x_{2}, a\right)>r_{0}$, it follows that

$$
\left(1+r_{0}\right) d\left(x_{2}, a\right) \geq r_{0}\left(1+d\left(x_{2}, a\right)\right)
$$

Hence, we have

$$
\begin{aligned}
d_{a}\left(x_{1}, x_{2}\right) & =\frac{d\left(x_{1}, x_{2}\right)}{\left[1+d\left(a, x_{1}\right)\right]\left[1+d\left(a, x_{2}\right)\right]} \geq \frac{c^{\prime} r}{\left[1+d\left(a, x_{1}\right)\right]\left[1+d\left(a, x_{2}\right)\right]} \\
& \geq \frac{c^{\prime} d\left(x_{2}, a\right)}{\left[1+d\left(a, x_{2}\right)\right]} \frac{1}{\left[1+d\left(a, x_{1}\right)\right]} \geq c^{\prime} \frac{r_{0}}{1+r_{0}} d_{a}\left(x_{1}, \infty\right) .
\end{aligned}
$$

Therefore, $\ell_{d_{a}}\left(\gamma_{x_{1} y_{1}}\right) \leq \frac{K^{4}\left(1+r_{0}\right)}{c^{\prime} r_{0}} d_{a}\left(x_{1}, x_{2}\right)$. Similarly, we can show that $\ell_{d_{a}}\left(\gamma_{x_{2} y_{2}}\right) \leq$ $\frac{K^{4}\left(1+r_{0}\right)}{c^{\prime} r_{0}} d_{a}\left(x_{1}, x_{2}\right)$.

We next obtain a bound for $\ell_{d_{a}}\left(\gamma_{y_{1} \infty}^{1}\right)$. By quasiconvexity, $\ell\left(\gamma_{a y_{1}}\right) \leq C d\left(a, y_{1}\right)$, where $\gamma_{a y_{1}}$ is the subcurve of $\gamma_{a \infty}^{1}$ ending at $y_{1}$. Let us choose a sequence of points $\left\{z_{n}\right\}_{n}$ with with $z_{0}=y_{1}$ and $z_{n} \in \gamma_{a \infty}^{1}$ such that $\ell\left(\gamma_{a \infty \mid\left[a, z_{n}\right]}^{1}\right)=2^{n} \ell\left(\gamma_{a z_{0}}\right)$. For simplicity, we denote by $\gamma_{z_{n} z_{n+1}}$ the subcurve of $\gamma_{a \infty}^{1}$ joining $z_{n}$ and $z_{n+1}$. For $w \in$ $\gamma_{z_{n} z_{n+1}}$, we have $\gamma_{w a} \subset \gamma_{z_{n+1} a}$, and hence by the base-point quasiconvexity of $\gamma_{a \infty}^{1}$,

$$
d(w, a) \geq \frac{1}{C} \ell\left(\gamma_{w a}\right) \geq \frac{1}{C} d\left(z_{n}, a\right) \geq \frac{1}{C^{2}} \ell\left(\gamma_{a z_{n}}\right) \geq \frac{2^{n}}{C^{2}} \ell\left(\gamma_{a z_{0}}\right) \geq \frac{2^{n}}{C^{2}} d\left(a, y_{1}\right) .
$$

Hence,

$$
\begin{aligned}
\ell_{d_{a}}\left(\gamma_{z_{n} z_{n+1}}\right) & =\int_{\gamma_{z_{n} z_{n+1}}} \frac{d s}{[1+d(\gamma(s), a)]^{2}} \leq \frac{\ell\left(\gamma_{z_{n} z_{n+1}}\right)}{\left[1+\frac{2^{n}}{C^{2}} d\left(a, y_{1}\right)\right]^{2}} \\
& \leq \frac{2^{n} \ell\left(\gamma_{a z_{0}}\right)}{\left[1+\frac{2^{n}}{C^{2}} d\left(a, y_{1}\right)\right]^{2}} \leq \frac{2^{n} C d\left(a, y_{1}\right)}{\left[1+\frac{2^{n}}{C^{2}} d\left(a, y_{1}\right)\right]^{2}}
\end{aligned}
$$

Notice also that because $d\left(x_{1}, y_{1}\right) \leq 2 r \leq 4 K d\left(a, y_{1}\right)$,

$$
d\left(x_{1}, a\right) \leq d\left(x_{1}, y_{1}\right)+d\left(y_{1}, a\right) \leq(1+4 K) d\left(a, y_{1}\right) .
$$

Therefore,

$$
\ell_{d_{a}}\left(\gamma_{z_{n} z_{n+1}}\right) \leq \frac{2^{n} C d\left(a, y_{1}\right)}{\left[1+\frac{2^{n}}{C^{2}} d\left(a, y_{1}\right)\right]^{2}} \leq \frac{C^{3}(1+K)}{2^{n} d\left(a, x_{1}\right)}=\frac{C}{2^{n} d\left(a, x_{1}\right)}
$$


Summing up and using analogs of (3.2) and (3.3), with $x_{1}$ and $x_{2}$ switching roles, we get

$$
\begin{aligned}
\ell_{d_{a}}\left(\gamma_{y_{1} \infty}^{1}\right) & =\sum_{n} \ell_{d_{a}}\left(\gamma_{z_{n} z_{n+1}}\right) \leq \sum_{n} \frac{C}{2^{n} d\left(a, x_{1}\right)} \leq \frac{C}{d\left(a, x_{1}\right)} \\
& \leq \frac{C\left(r_{0}+1\right)}{r_{0}} \frac{1}{1+d\left(a, x_{1}\right)} \leq \frac{C\left(r_{0}+1\right)^{2}}{c^{\prime} r_{0}^{2}} d_{a}\left(x_{1}, x_{2}\right) .
\end{aligned}
$$

We then have that for some $C \geq 1, \ell_{d_{a}}\left(\gamma_{y_{1} \infty}^{1}\right)+\ell_{d_{a}}\left(\gamma_{x_{1} y_{1}}\right) \leq C d_{a}\left(x_{1}, x_{2}\right)$. Similarly, one can prove that $\ell_{d_{a}}\left(\gamma_{y_{2} \infty}^{2}\right)+\ell_{d_{a}}\left(\gamma_{x_{2} y_{2}}\right) \leq C d_{a}\left(x_{1}, x_{2}\right)$. Putting all these estimates together, we conclude that

$$
\ell_{d_{a}}(\gamma)=\ell_{d_{a}}\left(\gamma_{x_{1} y_{1}}\right)+\ell_{d_{a}}\left(\gamma_{y_{1} \infty}^{1}\right)+\ell_{d_{a}}\left(\gamma_{y_{2} \infty}^{2}\right)+\ell_{d_{a}}\left(\gamma_{x_{2} y_{2}}\right) \leq C d_{a}\left(x_{1}, x_{2}\right) .
$$

Case 2. $x_{1}$ and $x_{2}$ are in the same annulus $A(a, r / 2, r)$ for some $r>r_{0}$ and $d\left(x_{1}, x_{2}\right) \leq c^{\prime} r$. Note again that $x_{1}, x_{2}$ cannot be equal to $\infty$. By the quasiconvexity of $X$, we can find a $C_{q}$-quasiconvex curve $\gamma_{x_{1} x_{2}}$ connecting $x_{1}$ and $x_{2}$. Because $c^{\prime} \leq 1 /\left(4 C_{q}\right)$, we have that $\ell\left(\gamma_{x_{1} x_{2}}\right) \leq C_{q} d\left(x_{1}, x_{2}\right) \leq r / 4$ and $\gamma_{x_{1} x_{2}}$ is contained in the annulus $A\left(a, r / 4,2 C_{q} r\right)$. Suppose $w \in \gamma_{x_{1} x_{2}}$, so $d\left(w, x_{1}\right) \leq \ell\left(\gamma_{x_{1} x_{2}}\right) \leq r / 4$.

Then $d(w, a) \geq d\left(x_{1}, a\right)-d\left(w, x_{1}\right) \geq r / 4$. Therefore, we can estimate the length of $\gamma_{x_{1} x_{2}}$ under the sphericalized metric as follows:

$$
\begin{aligned}
\ell_{d_{a}}\left(\gamma_{x_{1} x_{2}}\right) & =\int_{\gamma_{x_{1}, x_{2}}} \frac{d s}{[1+d(\gamma(s), a)]^{2}} \leq \frac{\ell\left(\gamma_{x_{1} x_{2}}\right)}{(1+r / 4)^{2}} \leq \frac{16 C_{q} d\left(x_{1}, x_{2}\right)}{(1+r)^{2}} \\
& \leq \frac{16 C_{q} d\left(x_{1}, x_{2}\right)}{\left[1+d\left(x_{1}, a\right)\right]\left[1+d\left(x_{2}, a\right)\right]} \leq 16 C_{q} d_{a}\left(x_{1}, x_{2}\right) .
\end{aligned}
$$

Case 3. $x_{1}$ and $x_{2}$ are in different annuli. Then again we have that $x_{1}, x_{2} \neq \infty$. We re-name $x_{1}$ and $x_{2}$ if necessary so that $d\left(a, x_{1}\right)<d\left(a, x_{2}\right)$. If $2 d\left(a, x_{1}\right)>d\left(a, x_{2}\right)$ and $d\left(x_{1}, a\right)>r_{0}$, let $r=d\left(x_{2}, a\right)$ and we can apply Case 1 or Case 2 to prove that there exists a curve $\gamma_{x_{1} x_{2}}$ connecting $x_{1}$ to $x_{2}$ with $\ell_{d_{a}}\left(\gamma_{x_{1} x_{2}}\right) \leq C d_{a}\left(x_{1}, x_{2}\right)$. Furthermore, if $d\left(a, x_{1}\right) \leq r_{0}$, then we can, by the connectivity of $X$, find a point $x_{1}^{\prime}$ with $d\left(x_{1}^{\prime}, a\right)=r_{0}$, find a quasiconvex curve connecting $x_{1}^{\prime}$ to $x_{2}$, and concatenate that curve with the quasiconvex curve connecting $x_{1}$ to $x_{1}^{\prime}$, and obtain a quasiconvex curve connecting $x_{1}$ to $x_{2}$. So without loss of generality, we can also assume that $d\left(a, x_{1}\right) \geq r_{0}$. Thus we will assume that $2 r_{0} \leq 2 d\left(a, x_{1}\right) \leq d\left(a, x_{2}\right)$.

We first find estimates for $d_{a}\left(x_{1}, x_{2}\right)$ as follows:

$$
\begin{aligned}
d_{a}\left(x_{1}, x_{2}\right) & =\frac{d\left(x_{1}, x_{2}\right)}{\left[1+d\left(x_{1}, a\right)\right]\left[1+d\left(x_{2}, a\right)\right]} \leq \frac{d\left(x_{1}, a\right)+d\left(x_{2}, a\right)}{\left[1+d\left(x_{1}, a\right)\right]\left[1+d\left(x_{2}, a\right)\right]} \\
& \leq \frac{3}{2} \frac{d\left(x_{2}, a\right)}{\left[1+d\left(x_{1}, a\right)\right]\left[1+d\left(x_{2}, a\right)\right]} \leq \frac{3}{2} \frac{1}{1+d\left(x_{1}, a\right)} .
\end{aligned}
$$

Furthermore, since we can take $r_{0} \geq 1$ in the definition of radially star-like quasiconvexity, we also see that

$$
d\left(x_{1}, x_{2}\right) \geq d\left(x_{2}, a\right)-d\left(x_{1}, a\right) \geq d\left(x_{2}, a\right) / 2 \geq\left[1+d\left(x_{2}, a\right)\right] / 4 .
$$

Therefore,

$$
d_{a}\left(x_{1}, x_{2}\right) \geq \frac{1}{4} \frac{1}{1+d\left(x_{1}, a\right)} .
$$

Let $\gamma_{a \infty}^{1}$ be a base-point quasiconvex ray from $a$ to $\infty$ associated with $x_{1}$ via the radial star-like quasiconvexity of $X$, and let $y_{1}$ be a point on this ray linked to $x_{1}$ as 
in the definition of radial star-likeness. Similarly, let $\gamma_{a \infty}^{2}$ be a base-point quasiconvex ray from $a$ to $\infty$ associated with $x_{2}$ via the radial star-like quasiconvexity of $X$, and let $y_{2}$ be a point on this ray linked to $x_{2}$ as in the definition of radial star-likeness. We will show that the the concatenation of the four curves $\gamma_{x_{1} y_{1}}, \gamma_{y_{2} x_{2}}$, the subcurve $\beta_{1}$ of $\gamma_{a \infty}^{1}$ with end points $y_{1}$ and $\infty$, and the subcurve $\beta_{2}$ of $\gamma_{a \infty}^{2}$ with end points $y_{2}$ and $\infty$, forms the desired quasiconvex curve (with respect to the metric $d_{a}$ ) connecting $x_{1}$ to $x_{2}$.

First, consider the quasiconvex curve $\gamma_{x_{1} y_{1}}$ connecting $x_{1}$ to $y_{1}$ and lying in the annulus $A(a, r / K, K r)$, where $r=d\left(x_{1}, a\right)$, as stipulated in the definition of radial star-likeness. Then for each point $w$ on that curve, we know that $d(w, a) \geq r / K$. Therefore

$$
\begin{aligned}
\ell_{d_{a}}\left(\gamma_{x_{1} y_{1}}\right) & =\int_{\gamma_{x_{1} y_{1}}} \frac{1}{[1+d(w, a)]^{2}} d s(w) \leq \frac{\ell\left(\gamma_{x_{1} y_{1}}\right)}{[1+r / K]^{2}} \leq C \frac{r}{[1+r / K]^{2}} \\
& \leq C \frac{1}{1+r / K} \leq \frac{C}{1+d\left(x_{1}, a\right)} \leq C d_{a}\left(x_{1}, x_{2}\right) .
\end{aligned}
$$

We used (3.6) to obtain the last inequality in the above chain of inequalities.

Next, we consider the quasiconvex curve $\gamma_{y_{2} x_{2}}$ connecting $x_{2}$ and $y_{2}$ that lies in the annulus $A(a, R / K, K R)$ with $R=d\left(x_{2}, a\right)$. Then for each point $w$ in that curve, we know that $d(w, a) \geq R / K$. Hence

$$
\begin{aligned}
\ell_{d_{a}}\left(\gamma_{y_{2} x_{2}}\right) & =\int_{\gamma_{y_{2} x_{2}}} \frac{1}{[1+d(w, a)]^{2}} d s(w) \leq \frac{\ell\left(\gamma_{y_{2} x_{2}}\right)}{[1+R / K]^{2}} \leq C \frac{R}{[1+R / K]^{2}} \\
& \leq C \frac{1}{1+R / K} \leq \frac{C}{1+d\left(x_{2}, a\right)} \leq \frac{C}{1+d\left(x_{1}, a\right)} \leq C d_{a}\left(x_{1}, x_{2}\right) .
\end{aligned}
$$

We again used (3.6) to obtain the above last inequality.

We finally consider the curves $\beta_{1}$ and $\beta_{2}$, and set $r=d\left(a, x_{1}\right), R=d\left(a, x_{2}\right)$. For non-negative integers $i$ let $z_{1, i}$ be the first point at which $\beta_{1}$ intersects $X \backslash B\left(a, 2^{i} r\right)$, and let $z_{2, i}$ be the first point at which $\beta_{2}$ intersects $X \backslash B\left(a, 2^{i} R\right)$. These points break $\beta_{1}$ and $\beta_{2}$ up into sub-curves $\beta_{1, i}$ and $\beta_{2, i}, i=0,1, \cdots$. By the base-point quasiconvexity of $\gamma_{a \infty}^{1}$ (see Definition 3.1), whenever $w$ is a point in $\beta_{1, i}$, we must have $d(a, w) \geq 2^{i-1} r / K$. It follows that

$$
\ell_{d_{a}}\left(\beta_{1, i}\right)=\int_{\beta_{1, i}} \frac{1}{[1+d(w, a)]^{2}} d s(w) \leq C \frac{\ell\left(\beta_{1, i}\right)}{\left[1+2^{i} r\right]^{2}} .
$$

By the base-point quasiconvexity again, $\ell\left(\beta_{1, i}\right) \leq K d\left(z_{1, i}, a\right) \leq K 2^{i} r$. Note that $1 \leq r_{0} \leq d\left(a, x_{1}\right)=r$. Hence,

$$
\ell_{d_{a}}\left(\beta_{1}\right)=\sum_{i} \ell_{d_{a}}\left(\beta_{1, i}\right) \leq C \sum_{i} \frac{2^{i} r}{\left[1+2^{i} r\right]^{2}} \leq C \sum_{i} \frac{2^{i} r}{4^{i} r^{2}} \leq \frac{C}{r} \leq \frac{C}{1+d\left(x_{1}, a\right)}
$$

Therefore, using (3.6), we obtain

$$
\ell_{d_{a}}\left(\beta_{1}\right) \leq C d_{a}\left(x_{1}, x_{2}\right) .
$$

A similar argument for $\beta_{2}$, with $r$ replaced by $R$, gives

$$
\ell_{d_{a}}\left(\beta_{2}\right) \leq \frac{C}{1+R}
$$

Because in Case 3 we have $r<R$, we have the desired inequality $\ell_{d_{a}}\left(\beta_{2}\right) \leq C d_{a}\left(x_{1}, x_{2}\right)$ as well. Thus the concatenated curve has $d_{a}$-metric length at most $4 C d_{a}\left(x_{1}, x_{2}\right)$, yielding the desired quasiconvex curve. 
Case 4. $x_{1}=\infty \neq x_{2}$. If $d\left(x_{2}, a\right) \leq r_{0}$, since $X$ is unbounded, there exists $x^{\prime}$ with $d\left(x^{\prime}, a\right)=r_{0}$. By the radially star-like quasiconvexity property and (3.7) (which is valid for all base-point quasiconvex rays and all points on those rays), there is $\gamma_{x^{\prime} \infty}$ such that $\ell_{d_{a}}\left(\gamma_{x^{\prime} \infty}\right) \leq C d_{a}\left(x^{\prime}, \infty\right)$. In addition, since $x_{2}, x^{\prime} \in \bar{B}\left(a, r_{0}\right)$, we have a $C_{q^{-}}$-quasiconvex curve $\gamma_{x_{2} x^{\prime}}$ with respect to $(X, d)$. By the argument at the beginning of this proof, we can see that $\gamma_{x_{2} x^{\prime}}$ is also quasiconvex with respect to $\left(\dot{X}, d_{a}\right)$, so

$$
\ell_{d_{a}}\left(\gamma_{x_{2} x^{\prime}}\right) \leq C d_{a}\left(x^{\prime}, x_{2}\right) \leq C d_{a}\left(x^{\prime}, \infty\right)+d_{a}\left(x_{2}, \infty\right) .
$$

Moreover, we have

$$
d_{a}\left(x^{\prime}, x_{2}\right)=\frac{d\left(x^{\prime}, x_{2}\right)}{\left[1+d\left(x^{\prime}, a\right)\right]\left[1+d\left(x_{2}, a\right)\right]} \leq \frac{1}{1+d\left(x^{\prime}, a\right)}+\frac{1}{1+d\left(x_{2}, a\right)} \leq 2 d_{a}\left(x_{2}, \infty\right),
$$

where the last inequality follows the fact that

$$
d_{a}\left(x_{2}, \infty\right)=\frac{1}{1+d\left(x_{2}, \infty\right)} \leq \frac{1}{1+d\left(x^{\prime}, \infty\right)}=d_{a}\left(x^{\prime}, \infty\right) .
$$

Therefore, when we concatenate the curves $\gamma_{x_{2} x^{\prime}}$ and $\gamma_{x^{\prime} \infty}$ to obtain a curve $\gamma_{x_{2} \infty}$ such that $\ell_{d_{a}}\left(\gamma_{x_{2} \infty}\right) \leq C d\left(x_{2}, \infty\right)$.

If $d\left(x_{2}, a\right) \geq r_{0}$, by the radially star-like quasiconvexity property, we have a base-point quasiconvex ray $\gamma_{a \infty}$ associated to $x_{2}$, and $y_{2}$ be the point on the ray connected to $x_{2}$. By (3.1) and (3.7), we have $\ell_{d_{a}}\left(\gamma_{x_{2} y_{2}}\right) \leq C d_{a}\left(x_{2}, \infty\right)$ and $\ell_{d_{a}}\left(\gamma_{y_{2} \infty}\right) \leq$ $C d_{a}\left(x_{2}, \infty\right)$. Therefore, we have shown the quasiconvexity of the metric space $\left(\dot{X}, d_{a}\right)$.

Remark 3.5. Example 3.7 will show that if we remove the hypothesis of radially star-like quasiconvexity, the previous theorem would be false. Notice also that quasiconvexity cannot be removed either. Let $X$ be a subspace of $\mathbf{R}^{2}$ given by $X=([0, \infty) \times\{1\}) \cup([0, \infty) \times\{-1\}) \cup(\{0\} \times[-1,1])$ together with the inherited Euclidean metric is radially star-like quasiconvex, not quasiconvex, and $\left(\dot{X}, d_{a}\right)$ is not quasiconvex as well.

As a consequence of Theorem 3.4 we obtain that $\infty$-Poincaré inequality is also preserved under sphericalization for radially star-like quasiconvex spaces.

Theorem 3.6. Let $(X, d, \mu)$ be a complete metric space endowed with a doubling measure $\mu$ and supporting an $\infty$-Poincaré inequality. Let $a \in X$ be a base point on $X$, and assume $(X, d)$ is $K$-radially star-like quasiconvex with respect to a for some $K \geq 2$. Then $\left(\dot{X}, d_{a}, \mu_{a}\right)$ also supports an $\infty$-Poincaré inequality.

Proof. First notice that the doubling property of $\mu_{a}$ has been shown in [22, Proposition 3.2.2]. Then, according to Theorem 2.1 we have to prove that there is a constant $C \geq 1$ such that, for every null set $N$ of $\dot{X}$, and for every pair of points $x, y \in \dot{X}$ there is a $C$-quasiconvex path $\gamma$ in $\dot{X}$ connecting $x$ to $y$ with $\gamma \notin \Gamma_{N}^{+}$. Given $N \subset X$, we have $\mu(N)=0$ if and only if $\mu_{a}(N)=0$ (when we consider $N$ as a subset of $\dot{X})$. Therefore, in the proof, we will not distinguish the null set $N \subset X$ and $N \subset \dot{X}$.

Without loss of generality, assume $x \neq \infty$ and $d_{a}(y, \infty) \leq d_{a}(x, \infty)$. Since $d_{a}(y, \infty) \leq d_{a}(x, \infty)$, it follows that $d(x, a) \leq d(y, a)$. As in the proof of Theorem 3.4, we can assume also that $d(y, a) \geq 2 r_{0}$ and $d(x, a) \geq r_{0} \geq 1$. If $d(y, a)<2 r_{0}$, then as in the first paragraph of the proof of Theorem 3.4, we see by the biLipschitz equivalence of the two metrics $d$ and $d_{a}$ on $B\left(a, 2 C_{q} r_{0}\right)$ and using the fact that $(X, d)$ supports an $\infty$-Poincaré inequality, that there is a quasiconvex curve $\gamma_{x y}$ in 
$B\left(a, 2 C_{q} r_{0}\right)$ connecting $x$ to $y$ (quasiconvex with respect to the metric $d$ and hence with respect to the metric $d_{a}$ ) such that $\gamma_{x y}$ does not belong to $\Gamma_{N}^{+}$. Thus in the remainder of this proof we will assume that $d(y, a) \geq 2 r_{0}$. If $d(y, a) \geq 2 r_{0}$ and $d(x, a)<r_{0}$ then we can find $x^{\prime}$ such that $d\left(x^{\prime}, a\right)=r_{0}$ and argue as before to find a quasiconvex curve $\gamma_{x x^{\prime}}$ connecting $x$ and $x^{\prime}$ with respect to the metric $d_{a}$ that is not in $\Gamma_{N}^{+}$. Then we can take the concatenation of $\gamma_{x x^{\prime}}$ and $\gamma_{x^{\prime} y}$, where $\gamma_{x^{\prime} y}$ will be constructed below.

Since $(X, d, \mu)$ is a complete metric space with a doubling measure $\mu$ and supports an $\infty$-Poincaré inequality, it is in particular $C^{\prime}$-quasiconvex for some $C^{\prime} \geq 1$, so $\dot{X}$ is also quasiconvex with respect to the metric $d_{a}$ by Theorem 3.4. Therefore, there is a quasiconvex curve $\gamma$ with respect to the metric $d_{a}$ that connects $x$ and $y$. If $\gamma$ intersects $B\left(a, r_{0}\right)$, let $p, q$ be the first and last time $\gamma$ intersects the sphere $\left\{z \in X: d(z, a)=r_{0}\right\}$, and then by the fact that $d_{a}, d$ are biLipschitz equivalent on $B\left(a, 2 C r_{0}\right)$ and that $(X, d)$ supports an $\infty$-Poincaré inequality, we can find a curve $\beta$ in $B\left(a, C r_{0}\right)$ that is quasiconvex both with respect to $d$ and $d_{a}$, and $\beta \notin \Gamma_{N}^{+}$, with end points $p, q$, and we can replace the segment of $\gamma$ with end points $p, q$ with the curve $\beta$. Hence we only have to concern ourselves with the part of $\gamma$ that lies outside the closed ball $\bar{B}\left(a, r_{0}\right)$. We can decompose that part of $\gamma$ into subcurves $\gamma_{i}$, $i \in I \subset \mathbf{N} \cup\{\infty\}$, so that for each $i$ the sub-curve $\gamma_{i}$ lies in an annulus $A\left(a, R_{i}, 4 R_{i}\right)$, with $R_{i}=2^{j_{i}} d(x, a) \geq r_{0}$ for some $j_{i} \in \mathbf{Z}$, and $\gamma$ is the concatenation of these curves. Note that it is possible to have $j_{i}=j_{l}$ even if $i \neq l$.

Let $x_{i}, y_{i}$ be the end points of $\gamma_{i}$. We next choose a finite chain of points $x_{i, 1}=$ $x_{i}, x_{i, 2}, \cdots, x_{i, N_{i}}=y_{i} \in \gamma_{i}$ such that for each $j=1, \cdots, N_{i}$,

$$
d\left(x_{i, j}, x_{i, j+1}\right) \leq \frac{R_{i}}{2 C^{\prime}} .
$$

Since $(X, d, \mu)$ supports an $\infty$-Poincaré inequality, there exists $\beta_{i, j}$ connecting $x_{i, j}$ and $x_{i, j+1}$ with $\ell\left(\beta_{i, j}\right) \leq C^{\prime} d\left(x_{i, j}, x_{i, j+1}\right) \leq R_{i} / 2$, and $\beta_{i, j} \notin \Gamma_{N}^{+}$.

Then, for every $w \in \beta_{i, j}$, we have $d(w, a)>R_{i}-\frac{R_{i}}{2}=\frac{R_{i}}{2}$ and so $1+d(w, a)>\frac{R_{i}}{2}$. Furthermore, because $R_{i} \geq r_{0} \geq 1$ we have that $1+d\left(x_{i, j}, a\right) \leq R_{i}+4 R_{i}=5 R_{i}$ and so

Hence

$$
d_{a}\left(x_{i, j}, x_{i, j+1}\right)=\frac{d\left(x_{i, j}, x_{i, j+1}\right)}{\left[1+d\left(x_{i, j}, a\right)\right]\left[1+d\left(x_{i, j+1}, a\right)\right]} \geq \frac{d\left(x_{i, j}, x_{i, j+1}\right)}{25 R_{i}^{2}} .
$$

$$
\begin{aligned}
\ell_{d_{a}}\left(\beta_{i, j}\right) & =\int_{\beta_{i, j}} \frac{1}{[1+d(w, a)]^{2}} d s(w) \leq \int_{0}^{\ell\left(\beta_{i, j}\right)} \frac{d s}{\left[R_{i} / 2\right]^{2}} \\
& \leq \frac{4 C^{\prime} d\left(x_{i, j}, x_{i, j+1}\right)}{R_{i}^{2}} \leq C d_{a}\left(x_{i, j}, x_{i, j+1}\right) .
\end{aligned}
$$

Furthermore, since $x_{i, j}, x_{i, j+1}$ are the end points of quasiconvex curves $\beta_{i, j}$ and these points lie in $\gamma_{i}$, we also have

$$
\sum_{j=1}^{N_{i}} d_{a}\left(x_{i, j}, x_{i, j+1}\right) \leq C \ell_{d_{a}}\left(\gamma_{i}\right)
$$

Setting $\beta_{i}$ to be the concatenation of the quasiconvex curves $\beta_{i, j}, j=1, \cdots, N_{i}$ and summing over $j$ we obtain

$$
\ell_{d_{a}}\left(\beta_{i}\right)=\sum_{j=1}^{N_{i}} \ell_{d_{a}}\left(\beta_{i, j}\right) \leq C \sum_{j=1}^{N_{i}} d_{a}\left(x_{i, j}, x_{i, j+1}\right) \leq C \ell_{d_{a}}\left(\gamma_{i}\right) .
$$


Clearly $\beta_{i} \notin \Gamma_{N}^{+}$. It follows that the concatenation $\gamma^{\prime}$ of the curves $\beta_{i}, i=1, \cdots, N$ (together with $\beta$, if it is the case), is a curve connecting $x$ to $y$ with

$$
\ell_{d_{a}}\left(\gamma^{\prime}\right) \leq C \ell_{d_{a}}(\gamma) \leq C d_{a}(x, y)
$$

with $\gamma^{\prime} \notin \Gamma_{N}^{+}$.

The following example gives a quasiconvex metric measure space endowed with a doubling measure, which is not radially star-like quasiconvex, supporting an $\infty$ Poincaré inequality but whose sphericalized space is not quasiconvex so in particular does not support an $\infty$-Poincaré inequality.

Example 3.7. Consider a length space $X \subset \mathbf{R}^{2}$ (in fact it is a tree) with initial point $a=(0,0) \in X$, constructed in the following way. Let $[0, \infty) \times\{0\}$ be a geodesic ray and select the points $x_{i}, i \geq 0$ from $[0, \infty) \times\{0\}$ such that $d\left(x_{i}, a\right)=2^{2^{i}}$. We can also take an i-th branch emanating from $x_{i}$, with end point $y_{i}=\left(2^{2^{i}}, 2^{2^{i+1}}\right)$. Then we have $d\left(x_{i}, y_{i}\right)=2^{2^{i+1}}$. So

$$
X=[0, \infty) \times\{0\} \cup \bigcup_{i \in \mathbf{N}}\left\{2^{2^{i}}\right\} \times\left[0,2^{2^{i+1}}\right],
$$

see Figure 2. In what follows $\left[x_{i}, y_{i}\right]$ will denote the segment in $\mathbf{R}^{2}$ connecting $x_{i}$ to $y_{i}$. Since $X$ is a length space, it is in particular quasiconvex. We now endow $X$ with the 1-dimensional Hausdorff measure $\mu=\mathcal{H}^{1}$, which is doubling. In fact, it can be shown that $(X, d, \mu)$ is Ahlfors 1-regular.

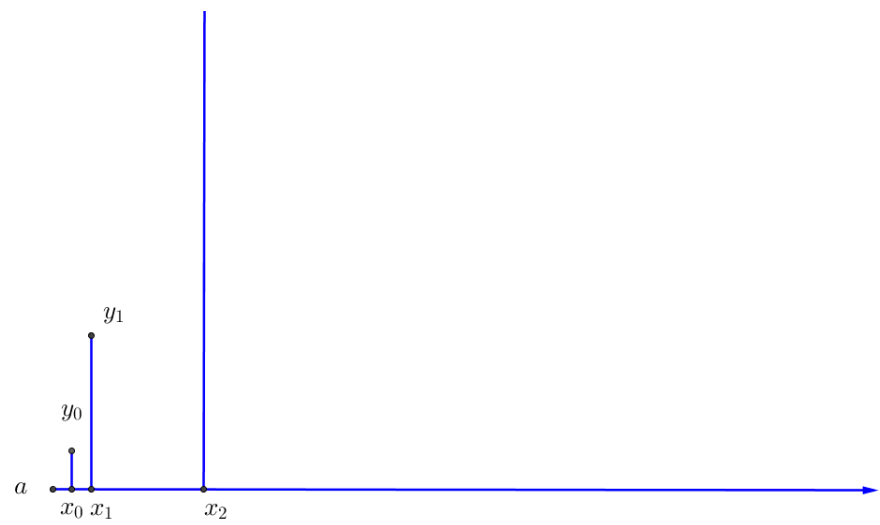

Figure 2. A metric tree which is not radially star-like quasiconvex.

Notice also that the $i$-th branch of the tree has length $2^{2^{i+1}}$, whereas the distance between $a$ and $x_{i}$ is only $2^{2^{i}}$, so the ratio between $d\left(x_{i}, y_{i}\right)$ and $d\left(x_{i}, a\right)$ would tend to $\infty$ as $i$ tends to $\infty$, which violates the assumption of radially star-like quasiconvexity. On the other hand, $(X, d, \mu)$ supports an $\infty$-Poincaré inequality. Indeed, according to Theorem 2.1, it is enough to check that for any null set $N \subset X$ and $x, y \in X$, there is a $C$-quasiconvex path connecting $x$ to $y$, with $\gamma \notin \Gamma_{N}^{+}$. In this case, we can let $\gamma$ to be the geodesic connecting $x$ and $y$.

To finish, we will show that the sphericalized space $\left(\dot{X}, d_{a}\right)$ is not quasiconvex, so in particular it does not support an $\infty$-Poincaré inequality. Suppose $i<j$. Let $y_{i}$ and $y_{j}$ be the end points of the $i$-th branch and $j$-th branch respectively. We have that

$$
d\left(y_{i}, y_{j}\right)=d\left(y_{i}, x_{i}\right)+d\left(x_{i}, x_{j}\right)+d\left(x_{j}, y_{j}\right)=2^{2^{i+1}}+2^{2^{j}}-2^{2^{i}}+2^{2^{j+1}} \approx 2^{2^{j+1}}
$$

and

$$
d\left(a, y_{i}\right)=2^{2^{i}}+2^{2^{i+1}}, d\left(a, y_{j}\right)=2^{2^{j}}+2^{2^{j+1}} .
$$


Therefore,

$$
d_{a}\left(y_{i}, y_{j}\right) \approx \frac{2^{2^{j+1}}}{2^{2^{i+1}} 2^{2^{j+1}}}=\frac{1}{2^{2^{i+1}}}
$$

In order to estimate the length of the curve $\gamma$ connecting $y_{i}$ and $y_{j}$, we have to take into account that $\gamma \supset\left[y_{i}, x_{i}\right] \cup\left[x_{i}, x_{j}\right] \cup\left[x_{j}, y_{j}\right]$. Denoting by $\gamma_{i}=\left[x_{i}, y_{i}\right]$, we have that

$$
\begin{aligned}
\ell_{d_{a}}(\gamma) & \geq \ell_{d_{a}}\left(\gamma_{i}\right)+\ell_{d_{a}}\left(\gamma_{j}\right)=\int_{0}^{\ell\left(\gamma_{i}\right)} \frac{d s}{\left[1+d\left(\gamma_{i}(s), a\right)\right]^{2}}+\int_{0}^{\ell\left(\gamma_{j}\right)} \frac{d s}{\left[1+d\left(\gamma_{j}(s), a\right)\right]^{2}} \\
& =\int_{0}^{\ell\left(\gamma_{i}\right)} \frac{d s}{\left[1+s+2^{2^{i}}\right]^{2}}+\int_{0}^{\ell\left(\gamma_{j}\right)} \frac{d s}{\left[1+s+2^{\left.2^{j}\right]^{2}}\right.} \\
& =\frac{1}{1+2^{2^{i}}}-\frac{1}{1+2^{2^{i}}+2^{2^{i+1}}}+\frac{1}{1+2^{2^{j}}}-\frac{1}{1+2^{2^{j}}+2^{2^{j+1}}} \approx \frac{1}{2^{2^{i}}} .
\end{aligned}
$$

Then,

$$
\frac{\ell_{d_{a}}(\gamma)}{d_{a}\left(y_{i}, y_{j}\right)} \geq \frac{2^{2^{i+1}}}{C 2^{2^{i}}} \approx \frac{2^{2^{i}}}{C}, \text { for all } i \geq 0 .
$$

Therefore, we have shown that $\left(\dot{X}, d_{a}\right)$ is not quasiconvex.

\section{Preservation of quasiconvexity and $\infty$-Poincaré inequality under flattening: meridian-like quasiconvex spaces}

In this section we introduce a general class of (bounded) metric spaces, meridianlike quasiconvex spaces, that preserves quasiconvexity and $\infty$-Poincaré inequalities under the flattening process. Meridian-like quasiconvexity is the dual property of radially star-like quasiconvexity: radially star-like quasiconvex spaces become meridianlike quasiconvex after sphericalization, and conversely, we recover radially star-like quasiconvexity after flattening meridian-like quasiconvex spaces.

Definition 4.1. A (bounded) metric space is $K$-meridian-like quasiconvex with respect to a base point $c \in X$, if there exists a constant $K \geq 2$, a point $a \in X$ and a small radius $r_{0}>0$ such that for every $x \in A(c, r / 2, r)$ with $r<r_{0}$ there exist a double base-point quasiconvex curve $\gamma_{a c}$, a point $y \in \gamma_{a c}$ and a curve $\gamma_{x y} \subset A(c, r / K, K r)$ connecting $x$ to $y$ such that

$$
\ell\left(\gamma_{x y}\right) \leq K d(y, c) .
$$

By double base-point quasiconvex curve we mean that for any $z \in \gamma_{a c}, \ell\left(\gamma_{a z}\right) \leq$ $C d(a, z)$ and $\ell\left(\gamma_{c z}\right) \leq C d(c, z)$. Here $\gamma_{a z}$ and $\gamma_{c z}$ denote the subcurves of $\gamma_{a c}$ with end points $a$ and $z$ and $c$ and $z$ respectively.

Remark 4.2. The idea is to choose the point $a \in X$ (in Definition 4.1) in $A(c, R / 2, R)$ where $R=\sup _{z \in X} d(c, z)$. Additionally, when $0<r \ll R$ and $x \in$ $B(c, r)$, we have $d(x, a) \approx d(a, c)$. Indeed, for $x \in B(c, r)$, we have that

$$
\begin{aligned}
2 d(a, c) & >d(a, c)+d(x, c) \geq d(a, x) \geq d(a, c)-d(x, c) \\
& \geq d(a, c)-r \approx d(a, c) .
\end{aligned}
$$

Observe also that meridian-like quasiconvexity implies that $c$ is not a cut point of $X$, that is, $X \backslash\{c\}$ is necessarily connected.

The following lemma shows that, for a general class of metric spaces, annular quasiconvexity is stronger than meridian-like quasiconvexity. 
Lemma 4.3. Let $(X, d)$ be a bounded connected complete locally compact metric space which is annularly quasiconvex with respect to a point $c \in X$. Then $(X, d)$ is $K$-meridian-like quasiconvex with respect to $c$.

Proof. The proof is similar to the proof of Lemma 3.3. First observe that under the hypothesis of the Lemma, the space $X$ is quasiconvex (see proof of Lemma 3.3). Given $x \in A(c, r / 2, r)$, we can find quasiconvex curves $\gamma_{c x}$ and $\gamma_{x a}$ to connect $c$ to $x$ and $x$ to $a$ respectively. As done in the proof of Lemma 3.3, we now modify the two curves in each annulus in order to ensure the base-point quasicovexity property with respect to $a$ and $c$ respectively. After denoting the modified curves by $\gamma_{c x}^{\prime}$ and $\gamma_{x a}^{\prime}$, we can show that the concatenation $\gamma=\gamma_{c x}^{\prime} \cup \gamma_{x a}^{\prime}$ is a double base-point quasiconvex curve connecting $c$ and $a$, which exactly passes through $x$.

The next two lemmas show that radially star-like quasiconvexity and meridianlike quasiconvexity are dual properties, with duality given via the sphericalization/ flattening procedures.

Lemma 4.4. Let $(X, d)$ be an unbounded complete metric space. Let $a \in X$ be a base point on $X$, and assume $(X, d)$ is $K$-radially star-like quasiconvex with respect to the base point a for some $K \geq 2$. Then $\left(\dot{X}, d_{a}\right)$ is $K^{\prime}$-meridian-like quasiconvex with respect to $c=\infty$ for some $K^{\prime} \geq 2$.

Proof. For $x \in X$, we know that $d(x, a)>r_{0}$ if and only if $d_{a}(x, \infty)<1-\frac{r_{0}}{1+r_{0}}$. Let $x \in B_{a}\left(\infty, 1-\frac{r_{0}}{1+r_{0}}\right) \backslash\{\infty\}$. By the $K$-radially star-like quasiconvex property there exist a base-point quasiconvex ray $\gamma_{a \infty}$, a point $y \in \gamma_{a \infty}$ and a quasiconvex curve $\gamma_{x y}$ connecting $x$ to $y$ in the annulus $A(a, d(a, x) / K, K d(a, x))$ such that $\ell\left(\gamma_{x y}\right) \leq$ $K d(a, y)$. Notice that the sphericalization of the ray $\gamma_{a \infty}$ is a base-point quasiconvex curve in $\dot{X}$ connecting $c=\infty$ to $a$. Indeed, for each $z \in \gamma_{a \infty}$, we denote the segment of $\gamma_{a \infty}$ with end points $z$ and $\infty$ by $\gamma_{z \infty}$, and note that $\ell_{d_{a}}\left(\gamma_{z \infty}\right) \leq C d_{a}(z, \infty)$. We have that (we denote the subcurve of $\gamma_{a \infty}$ with end points $a$ and $w$ by $\gamma_{a w}$ in the following):

$$
\begin{aligned}
\ell_{d_{a}}\left(\gamma_{z \infty}\right) & =\int_{\gamma_{z \infty}} \frac{d s}{[1+d(\gamma(s), a)]^{2}} \leq \int_{\gamma_{z \infty}} \frac{d s}{\left[1+\ell\left(\gamma_{a \gamma(s)}\right) / K\right]^{2}} \leq K^{2} \int_{\ell\left(\gamma_{a z}\right)}^{\infty} \frac{d t}{(1+t)^{2}} \\
& \leq \frac{K^{2}}{1+\ell\left(\gamma_{a z}\right)} \leq \frac{K^{3}}{1+d(z, a)}=C d_{a}(z, \infty),
\end{aligned}
$$

where in the first inequality we have used the fact that $\gamma_{a \infty}$ is a base-point quasiconvex ray and the second inequality we have used the fact that $\gamma$ is arc-length parametrized.

Moreover, $\ell_{d_{a}}\left(\gamma_{x y}\right) \leq C d_{a}(y, c)$. Indeed, let $r=d(x, a)>r_{0}$. Because $r / K \leq$ $d(w, a) \leq K r$ for any $w \in \gamma_{x y}$ with $\ell\left(\gamma_{x y}\right) \leq K r$, we have that

$$
\ell_{d_{a}}\left(\gamma_{x y}\right)=\int_{\gamma_{x y}} \frac{d s}{(1+d(\gamma(s), a))^{2}} \leq \frac{K d(y, a)}{\left(1+\frac{r}{K}\right)^{2}} \leq \frac{K^{3}}{1+\frac{r}{K}} \leq \frac{K^{5}}{1+K r} \approx d_{a}(y, c) .
$$

To finish notice that an annulus $A(a, r / 2, r)$ in the original metric is transformed under sphericalization into another annulus comparable to an annulus $A\left(\infty, r^{\prime} / 2, r^{\prime}\right)$ in the sphericalized metric for some $r^{\prime}>0$.

Lemma 4.5. Let $(X, d)$ be a bounded complete metric space. Let $c \in X$ and assume $(X, d)$ is $K$-meridian-like quasiconvex with respect to the base point $c$ for some $K \geq 2$. Then $\left(X^{c}, d^{c}\right)$ is $K^{\prime}$-radially star-like quasiconvex with respect to the point $a \in X$ (as in Remark 4.2) for some $K^{\prime} \geq 2$. 
Proof. Because $X$ is $K$-meridian-like quasiconvex with respect to a base point $c \in$ $X$, there exist a constant $K \geq 2$, a point $a \in X$ and a small radius $r_{0}>0$ such that for every $x \in A(c, r / 2, r)$ and $r<r_{0}$, there exist a double base-point quasiconvex curve $\gamma_{a c}$ connecting $a$ to $c$, a point $y \in \gamma_{a c}$ and a curve $\gamma_{x y} \subset A(c, r / K, K r)$ connecting $x$ to $y$ such that $\ell\left(\gamma_{x y}\right) \leq K d(y, c)$.

Now, we can show that the double base-point quasiconvex curve $\gamma_{a c}$ is a basepoint quasiconvex ray after flattening. Let $z \in \gamma_{a c}$ and consider the subcurves $\gamma_{a z}$ and $\gamma_{z c}$ of $\gamma_{a c}$ with starting points $a, z$ and end points $z, c$ respectively. Since $\ell\left(\gamma_{c z}\right) \leq$ $K d(z, c)$ and $\ell\left(\gamma_{a z}\right) \leq K d(a, z)$ for any $z \in \gamma_{a c}$, it follows that

$$
\begin{aligned}
\ell_{d^{c}}\left(\gamma_{a z}\right) & =\int_{\gamma_{a z}} \frac{d s}{[d(\gamma(s), c)]^{2}} \leq \int_{\ell\left(\gamma_{z c}\right)}^{\ell\left(\gamma_{a c}\right)} \frac{K^{2} d s}{s^{2}} \leq C\left(\frac{1}{\ell\left(\gamma_{z c}\right)}-\frac{1}{\ell\left(\gamma_{a c}\right)}\right) \\
& \leq \frac{C \ell\left(\gamma_{a z}\right)}{\ell\left(\gamma_{z c}\right) \ell\left(\gamma_{a c}\right)} \leq \frac{C d(a, z)}{d(c, z) d(c, a)}=C d^{c}(a, z) .
\end{aligned}
$$

Next we prove that for $x, y$ and $\gamma_{x y}$ satisfying (4.1), we have $\ell_{d^{c}}\left(\gamma_{x y}\right) \leq C d^{c}(a, y)$, for some $C \geq 1$ depending on $K$. See that for $w \in \gamma_{x y}$ we have that $d(w, c) \geq r / K$ so if $r_{0}$ is small enough,

$$
\ell_{d^{c}}\left(\gamma_{x y}\right)=\int_{\gamma_{x y}} \frac{d s}{[d(\gamma(s), c)]^{2}} \leq \frac{C \ell\left(\gamma_{x y}\right)}{r^{2}} \leq \frac{C d(y, c)}{r^{2}} \leq \frac{C}{d(y, c)} \stackrel{(4.2)}{\leq} C d^{c}(a, y) .
$$

To finish, notice that an annulus $A(c, r / K, K r)$ in the original metric transforms under flattening into another annulus comparable to an annulus $A\left(a, r^{\prime} / K^{\prime}, K^{\prime} r^{\prime}\right)$ in the flattened metric for some $r^{\prime}>0$ that depends only on $r$ and $K$, with $K^{\prime}$ depending solely on $K$.

Theorem 4.6. Let $(X, d)$ be a bounded complete metric space. Let $c \in X$ be a base point on $X$, and assume $(X, d)$ is quasiconvex and $K$-meridian-like quasiconvex with respect the base point $c$ for some $K \geq 2$. Then $\left(X^{c}, d^{c}\right)$ is quasiconvex.

Recall that $\ell$ denotes the length of the original metric and $\ell_{d^{c}}$ denotes the length of the flattened metric.

Proof. Since $(X, d)$ is quasiconvex and meridian-like quasiconvex, by Lemma 4.5 we know that $\left(X^{c}, d^{c}\right)$ is radially star-like quasiconvex with respect to $a$ where $a$ is the second point associated with the notion of meridian-like quasiconvexity.

The idea for the proof of this theorem is similar to the one used in Theorem 3.4. Given $x_{1}, x_{2} \in X^{c}$, we have to prove that there exists a curve $\gamma$ connecting $x_{1}$ and $x_{2}$ such that $\ell_{d^{c}}(\gamma) \leq C d^{c}\left(x_{1}, x_{2}\right)$. Let $r_{0}$ be as in Definition 4.1.

We divide the proof into five cases: points that are in the same annulus and far away from each other (Case 1), points that are in the same annulus and close to each other (Case 2), points lying in different annuli (Case 3) and points lying outside the ball $B\left(c, r_{0}\right)$ (Case 4 ) and finally one point in the ball $B\left(c, r_{0}\right)$ and another point outside of $B\left(c, r_{0}\right)$ (Case 5$)$. In the proof, the annuli are considered with respect to the original metric $d$. $X$.

Fix $c^{\prime}>0$ such that $0<c^{\prime}<1 /\left(4 C_{q}\right)$ where $C_{q}$ is the quasiconvexity constant of

Case 1. $x_{1}, x_{2}$ are in the annulus $A(c, r / 2, r)$ for some $r<r_{0}$ and $d\left(x_{1}, x_{2}\right) \geq c^{\prime} r$. Then we can find two double base-point quasiconvex curves $\gamma_{a c}^{1}, \gamma_{a c}^{2}$ and points $y_{1} \in$ $\gamma_{a c}^{1}, y_{2} \in \gamma_{a c}^{2}$ and quasiconvex curves $\gamma_{x_{1} y_{1}}, \gamma_{x_{2} y_{2}} \subset A(c, r / K, K r)$ satisfying (4.1). We 
want to show that the concatenation $\gamma=\gamma_{x_{1} y_{1}} \cup \gamma_{y_{1} a}^{1} \cup \gamma_{a y_{2}}^{2} \cup \gamma_{y_{2} x_{2}}$ is a quasiconvex curve in the flattened metric.

Because $d\left(a, x_{1}\right), d\left(a, y_{1}\right), d\left(a, x_{2}\right), d\left(a, y_{2}\right)$ and $d\left(x_{1}, x_{2}\right)$ are all comparable to $r$, we have that

$$
d^{c}\left(a, x_{1}\right) \approx d^{c}\left(a, y_{1}\right) \approx d^{c}\left(a, x_{2}\right) \approx d^{c}\left(a, y_{2}\right) \approx d^{c}\left(x_{1}, x_{2}\right)
$$

Using (4.4), we can estimate $\ell_{d^{c}}\left(\gamma_{x_{1} y_{1}}\right)$ as follows:

$$
\ell_{d^{c}}\left(\gamma_{x_{1} y_{1}}\right) \leq C d^{c}\left(y_{1}, a\right) \leq C d^{c}\left(x_{1}, x_{2}\right) .
$$

Next, because $y_{1} \in B(c, K r)$, by (4.3) we have

$$
\ell_{d^{c}}\left(\gamma_{a y_{1}}\right) \leq C d^{c}\left(a, y_{1}\right) \leq C d^{c}\left(x_{1}, x_{2}\right) .
$$

Similar arguments give us estimates for $\ell_{d^{c}}\left(\gamma_{a y_{2}}\right)$ and $\ell_{d^{c}}\left(\gamma_{x_{2} y_{2}}\right)$ in terms of $d^{c}\left(x_{1}, x_{2}\right)$. Therefore, we have $\ell_{d^{c}}(\gamma) \leq C d^{c}\left(x_{1}, x_{2}\right)$.

Case 2. $x_{1}, x_{2}$ are in the same annulus $A(c, r / 2, r), r \leq r_{0}$, and $d\left(x_{1}, x_{2}\right) \leq c^{\prime} r$. By the quasiconvexity of $(X, d)$, we can find a $C_{q}$-quasiconvex curve $\gamma$ connecting $x_{1}, x_{2}$. Because $c^{\prime}<1 /\left(4 C_{q}\right)$, for $w \in \gamma$, we can get the estimate

$d(w, c) \geq d\left(x_{1}, c\right)-d\left(x_{1}, w\right) \geq d\left(x_{1}, c\right)-\ell(\gamma) \geq r / 2-C_{q} d\left(x_{1}, x_{2}\right) \geq r / 2-r / 4=r / 4$,

SO

$$
\ell_{d^{c}}(\gamma)=\int_{\gamma} \frac{d s}{[d(\gamma(s), c)]^{2}} \leq \frac{16 \ell(\gamma)}{r^{2}} \leq \frac{16 C_{q} d\left(x_{1}, x_{2}\right)}{d\left(x_{1}, c\right) d\left(x_{2}, c\right)} \leq C d^{c}\left(x_{1}, x_{2}\right) .
$$

Hence, we have proved quasiconvexity in Case 2 .

Case 3. $2 d\left(x_{1}, c\right) \leq d\left(x_{2}, c\right) \leq r_{0}$. By the definition of meridian-like quasiconvexity, we can choose $r_{0}$ as small as we need, and so we choose $0<r_{0}<d(a, c) / 2$. Then we have $d(a, c) \geq 2 r_{0}$, and $x_{1}, x_{2} \in \bar{B}\left(c, r_{0}\right)$, we have $d\left(a, x_{1}\right) \approx d(a, c)$ and $d\left(a, x_{2}\right) \approx d(a, c)$, and hence

$$
\begin{aligned}
d^{c}\left(x_{1}, x_{2}\right) & =\frac{d\left(x_{1}, x_{2}\right)}{d\left(x_{1}, c\right) d\left(x_{2}, c\right)} \geq \frac{d\left(x_{2}, c\right)-d\left(x_{1}, c\right)}{d\left(x_{1}, c\right) d\left(x_{2}, c\right)} \geq \frac{1}{d\left(x_{1}, c\right)}-\frac{1}{d\left(x_{2}, c\right)} \\
& \geq \frac{1}{2 d\left(x_{1}, c\right)} \geq C^{-1} \frac{d\left(x_{1}, a\right)}{d\left(x_{1}, c\right) d(a, c)}=C^{-1} d^{c}\left(x_{1}, a\right) .
\end{aligned}
$$

Similarly, we have $d^{c}\left(x_{2}, a\right) \leq C d^{c}\left(x_{1}, x_{2}\right)$. Therefore, as in Case 1, by (4.3) and (4.4),

$$
\begin{aligned}
& \ell_{d^{c}}\left(\gamma_{y_{1} a}^{1}\right) \leq C d^{c}\left(y_{1}, a\right) \leq C d^{c}\left(x_{1}, a\right) \leq C d^{c}\left(x_{1}, x_{2}\right), \\
& \ell_{d^{c}}\left(\gamma_{y_{1} a}^{2}\right) \leq C d^{c}\left(y_{2}, a\right) \leq C d^{c}\left(x_{2}, a\right) \leq C d^{c}\left(x_{1}, x_{2}\right), \\
& \ell_{d^{c}}\left(\gamma_{x_{1} y_{1}}\right) \leq C d^{c}\left(x_{1}, a\right) \leq C d^{c}\left(x_{1}, x_{2}\right) .
\end{aligned}
$$

Therefore

$$
\ell_{d^{c}}\left(\gamma_{x_{2} y_{2}}\right) \leq C d^{c}\left(x_{2}, a\right) \leq C d^{c}\left(x_{1}, x_{2}\right) .
$$

So the concatenation $\gamma=\gamma_{x_{1} y_{1}} \cup \gamma_{y_{1} a}^{1} \cup \gamma_{a y_{2}}^{2} \cup \gamma_{y_{2} x_{2}}$ is quasiconvex.

Case 4. If $x_{1}, x_{2} \notin B\left(c, r_{0}\right), d^{c}$ and $d$ are biLipschitz equivalent on $X \backslash \overline{B\left(c, r_{0}\right)}$. Indeed, because $r_{0}<d\left(x_{i}, c\right)<\operatorname{diam} X$ for $i=1,2$, we have

$$
\frac{d\left(x_{1}, x_{2}\right)}{(\operatorname{diam} X)^{2}} \leq \frac{d\left(x_{1}, x_{2}\right)}{d\left(x_{1}, c\right) d\left(x_{2}, c\right)}=d^{c}\left(x_{1}, x_{2}\right) \leq \frac{d\left(x_{1}, x_{2}\right)}{\left(r_{0}\right)^{2}} .
$$

Because $(X, d)$ is quasiconvex, we can find a $C_{q}$-quasiconvex curve $\gamma$ connecting $x_{1}, x_{2}$ with respect to the metric $d$. If $\gamma$ intersects $B\left(c, r_{0}\right)$, let $p, q$ be the first and last time that $\gamma$ intersects the sphere $\left\{z \in X: d(z, c)=r_{0}\right\}$. By the fact that $d^{c}$ and $d$ are biLipschitz equivalent on $X \backslash \overline{B\left(c, r_{0}\right)}$, the subcurves of $\gamma, \gamma_{x_{1} p}$ and $\gamma_{q x_{2}}$, connecting 
$x_{1}$ to $p$ and $q$ to $x_{2}$ respectively, are also quasiconvex with respect to the metric $d^{c}$. Using either Case 1 or Case 2 (depending on the distance between $p$ and $q$ ), we now find a quasiconvex curve $\beta$ with respect to the metric $d^{c}$ and replace the segment of $\gamma$ with end points $p, q$ with the curve $\beta$. The concatenation of the curves $\gamma_{x_{1} p}, \beta$ and $\gamma_{q x_{2}}$ is a quasiconvex curve with respect to the metric $d^{c}$ connecting $x_{1}$ and $x_{2}$.

Case 5. $x_{1} \in B\left(c, r_{0}\right), x_{2} \notin B\left(c, r_{0}\right)$. Let $d\left(x_{1}, c\right)=r<r_{0} \leq d\left(x_{2}, c\right)$. If $d\left(x_{1}, x_{2}\right) \leq c^{\prime} r_{0}$, then as in Case 2 , we can find a quasiconvex curve $\gamma_{x_{1} x_{2}}$ connecting $x_{1}$ to $x_{2}$. Since $c^{\prime}<1 /\left(4 C_{q}\right)$, for $z \in \gamma_{x_{1} x_{2}}, d(z, c) \geq r_{0}-C_{q} c^{\prime} r_{0} \geq r_{0} / 2$, and so

$$
\ell_{d^{c}}\left(\gamma_{x_{1} x_{2}}\right) \leq \int_{\gamma_{x_{1} x_{2}}} \frac{d s}{[d(\gamma(s), c)]^{2}} \leq \frac{4 \ell\left(\gamma_{x_{1} x_{2}}\right)}{r_{0}^{2}} \leq \frac{4 C_{q} d\left(x_{1}, x_{2}\right)}{r_{0}^{2}},
$$

and

$$
d^{c}\left(x_{1}, x_{2}\right)=\frac{d\left(x_{1}, x_{2}\right)}{d\left(x_{1}, c\right) d\left(x_{2}, c\right)} \geq \frac{d\left(x_{1}, x_{2}\right)}{\left(2 r_{0}\right)\left(c^{\prime} r_{0}\right)} .
$$

On the other hand, if $d\left(x_{1}, x_{2}\right) \geq c^{\prime} r_{0}$, let $x^{\prime} \in X^{c}$ with $d\left(x^{\prime}, c\right)=r_{0}$. Therefore, by Case 1 or Case 2 (depending on the distance between $x_{1}$ and $x^{\prime}$ ), we can find a quasiconvex curve $\gamma_{x_{1} x^{\prime}}$ with respect to the metric $d^{c}$, and by case 4 , we can find a quasiconvex curve $\gamma_{x^{\prime} x_{2}}$ with respect to the metric $d^{c}$ with

$$
\ell_{d^{c}}\left(\gamma_{x_{1} x^{\prime}}\right) \leq C d^{c}\left(x_{1}, x^{\prime}\right), \ell_{d^{c}}\left(\gamma_{x^{\prime} x_{2}}\right) \leq C d^{c}\left(x^{\prime}, x_{2}\right) .
$$

Finally, we only need to show $d^{c}\left(x_{1}, x^{\prime}\right)+d^{c}\left(x^{\prime}, x_{2}\right) \leq C d^{c}\left(x_{1}, x_{2}\right)$.

Notice that

$$
d^{c}\left(x_{1}, x^{\prime}\right)=\frac{d\left(x_{1}, x^{\prime}\right)}{d\left(x_{1}, c\right) d\left(x^{\prime}, c\right)}, d^{c}\left(x_{2}, x^{\prime}\right)=\frac{d\left(x_{2}, x^{\prime}\right)}{d\left(x_{2}, c\right) d\left(x^{\prime}, c\right)}, d^{c}\left(x_{1}, x_{2}\right)=\frac{d\left(x_{1}, x_{2}\right)}{d\left(x_{2}, c\right) d\left(x^{\prime}, c\right)}
$$

and we have $d\left(x^{\prime}, c\right)=r_{0}, d\left(x_{1}, x_{2}\right) \geq c^{\prime} r_{0}, d\left(x_{1}, c\right)<r_{0} \leq d\left(x_{2}, c\right)$, so we can get

$$
\frac{d\left(x_{2}, x^{\prime}\right)}{d\left(x_{2}, c\right) d\left(x^{\prime}, c\right)} \leq \frac{d\left(x_{2}, x_{1}\right)+d\left(x_{1}, x^{\prime}\right)}{d\left(x_{2}, c\right) r_{0}} \leq \frac{d\left(x_{1}, x_{2}\right)}{d\left(x_{2}, c\right) d\left(x_{1}, c\right)}+\frac{d\left(x_{1}, x^{\prime}\right)}{d\left(x_{1}, c\right) r_{0}} \text {. }
$$

Therefore, we only need to show that

$$
\frac{d\left(x_{1}, x^{\prime}\right)}{d\left(x_{1}, c\right) r_{0}} \leq C \frac{d\left(x_{1}, x_{2}\right)}{d\left(x_{2}, c\right) d\left(x_{1}, c\right)}
$$

that is, $d\left(x_{1}, x^{\prime}\right) d\left(x_{2}, c\right) \leq C d\left(x_{1}, x_{2}\right) d\left(x^{\prime}, c\right)$. Since $d\left(x_{1}, x^{\prime}\right) \leq d\left(x_{1}, c\right)+d\left(x^{\prime}, c\right) \leq 2 r_{0}$ and $c^{\prime} r_{0} \leq d\left(x_{1}, x_{2}\right)$ we can obtain

$d\left(x_{1}, x^{\prime}\right) d\left(x_{2}, c\right) \leq d\left(x_{1}, x^{\prime}\right)\left[d\left(x_{1}, x_{2}\right)+d\left(x_{1}, c\right)\right] \leq 2 r_{0} d\left(x_{1}, x_{2}\right)+2 r_{0}^{2} \leq 2 C r_{0} d\left(x_{1}, x_{2}\right)$.

Combining the arguments above, we have proved that $d^{c}\left(x_{1}, x^{\prime}\right)+d^{c}\left(x^{\prime}, x_{2}\right) \leq d^{c}\left(x_{1}\right.$, $\left.x_{2}\right)$.

As a consequence of Theorem 4.6 we obtain that $\infty$-Poincaré inequality is also preserved under flattening for meridian-like quasiconvex spaces.

Theorem 4.7. Let $(X, d, \mu)$ be a complete bounded metric space endowed with a doubling measure $\mu$ and supporting an $\infty$-Poincaré inequality. Let $c \in X$ be a base point on $X$, and assume $(X, d)$ is $K$-meridian-like quasiconvex with respect to the base point $c$ for some $K \geq 2$. Then $\left(X^{c}, d^{c}, \mu^{c}\right)$ also supports an $\infty$-Poincaré inequality.

Proof. The proof is similar to the proof of Theorem 3.6. Since $(X, d, \mu)$ is a complete metric space with a doubling measure $\mu$ and supports and $\infty$-Poincaré inequality, then it is $C^{\prime}$-quasiconvex for some $C^{\prime} \geq 1$, so by Theorem $4.6,\left(X^{c}, d^{c}\right)$ is quasiconvex and there is quasiconvex curve $\gamma$ with respect to the metric $d^{c}$ connecting 
$x$ and $y$. Notice that the doubling property of $\mu^{c}$ has been shown in [22, Proposition 4.2.1.]. Now, according to Theorem 2.1 we need to prove that there is a constant $C \geq 1$ such that, for every null set $N$ of $X^{c}$, and for every pair of points $x, y \in X^{c}$ there is a $C$-quasiconvex path $\gamma$ in $X^{c}$ connecting $x$ to $y$ with $\gamma \notin \Gamma_{N}^{+}$. Given $N \subset X$ with $c \notin N$, we have $\mu(N)=0$ if and only if $\mu^{c}(N)=0$ (if we consider $N$ as a subset of $X^{c}$ ). Therefore, in the proof, we will not distinguish the null set $N \subset X$ and $N \subset X^{c}$.

Without loss of generality, we assume $d(x, c) \leq d(y, c)$. Let $x \in A\left(c, 2^{-k} R\right.$, $\left.2^{-k+1} R\right), y \in A\left(c, 2^{-j} R, 2^{-j+1} R\right)$, for some $j \leq k$, where $R=\sup _{z \in X} d(c, z)$.

First, we want to show that there exists $m \geq k+2$ with $\gamma \cap B\left(c, 2^{-m} R\right)=\emptyset$. Assume $\gamma \cap B\left(c, 2^{-m} R\right) \neq \emptyset$, for arbitrary big $m \in \mathbf{N}$. Notice that

$$
\ell_{d^{c}}(\gamma) \leq C d^{c}(x, y)=C \frac{d(x, y)}{d(x, c) d(y, c)} \leq C \frac{2 d(y, c)}{d(x, c) d(y, c)} \leq 2 C 2^{k} / R
$$

Let $\gamma_{m} \subset A\left(c, 2^{-m+1} R, 2^{-m+2} R\right)$ be a subcurve of $\gamma$ with end points $z_{m}, z_{m}^{\prime}$ such that $d\left(z_{m}, c\right)=2^{-m+1} R, d\left(z_{m}^{\prime}, c\right)=2^{-m+2} R$, then

$$
\ell_{d^{c}}\left(\gamma_{z_{m} z_{m}^{\prime}}\right)=\int_{0}^{\ell\left(\gamma_{z_{m} z_{m}^{\prime}}\right)} \frac{d s}{(d(c, \gamma(s)))^{2}} \geq \frac{\ell\left(\gamma_{z_{m} z_{m}^{\prime}}\right)}{\left(2^{-m+2} R\right)^{2}} \geq \frac{d\left(z_{m}, z_{m}^{\prime}\right)}{\left(2^{-m+2} R\right)^{2}} \geq 2^{m-3} / R
$$

Combining (4.5) and (4.6), we can see that $m$ cannot be too large.

Now, we can choose a sequence of points $x_{0}=x, x_{1}, \cdots, x_{N}=y$ in $\gamma$ and construct a collection of quasiconvex curves $\gamma_{1}, \gamma_{2}, \ldots, \gamma_{N}$ with respect to the metric $d^{c}$ such that the end points of $\gamma_{i}$ are $x_{i-1}$ and $x_{i}$ for each $1 \leq i \leq N$. We will construct the curve $\gamma_{i}$ by induction. First notice that $x_{0}=x \in A\left(c, 2^{-k} R, 2^{-k+1} R\right)$ and denote $j_{0}=k$. If $\ell\left(\gamma_{x_{0} y}\right) \leq 2^{-j_{0}} R /\left(2 C^{\prime}\right)$, we just let $x_{1}=y$. If not, let $x_{1} \in \gamma$ be a point such that the length of the subcurve of $\gamma$ connecting $x_{0}$ and $x_{1}$ (denoted by $\left.\gamma_{x_{0} x_{1}}\right)$ has length $\ell\left(\gamma_{x_{0} x_{1}}\right)=2^{-j_{0}} R /\left(2 C^{\prime}\right)$.

Notice that $\ell\left(\gamma_{x_{0} x_{1}}\right) \leq 2^{-j_{0}} R /\left(2 C^{\prime}\right)$, and for $z \in \gamma_{x_{0} x_{1}}$

$$
d(z, c) \geq d\left(x_{0}, c\right)-\ell\left(\gamma_{x_{0} z}\right) \geq 2^{-j_{0}} R-2^{-j_{0}} R /\left(2 C^{\prime}\right) \geq 2^{-j_{0}} R / 2 .
$$

Similarly, we obtain that $d(z, c) \leq 2^{-j_{0}+2} R$. Therefore, we can see that $\gamma_{x_{0} x_{1}} \subset$ $A\left(c, 2^{-j_{0}-1} R, 2^{-j_{0}+2} R\right)$. Since $x_{1}$ has been chosen, we take $j_{1}$ as the integer such that $2^{-j_{1}} R \leq d\left(x_{1}, c\right)<2^{-j_{1}+1} R$. Since $(X, d, \mu)$ supports an $\infty$-Poincaré inequality, we can take a $C^{\prime}$-quasiconvex curve $\gamma_{1} \notin \Gamma_{N}^{+}$connecting $x_{0}$ and $x_{1}$. On the other hand, since $d\left(x_{0}, x_{1}\right) \leq \ell\left(\gamma_{x_{0} x_{1}}\right) \leq 2^{-j_{0}} R /\left(2 C^{\prime}\right)$, we have $\ell\left(\gamma_{1}\right) \leq C^{\prime} d\left(x_{0}, x_{1}\right) \leq 2^{-j_{0}} R / 2$, similar to the argument above, we can show that for $z \in \gamma_{1}$, we have $2^{-j_{0}-1} R \leq$ $d(z, c) \leq 2^{-j_{0}+2} R$. Hence, we can obtain $\gamma_{1} \subset A\left(c, 2^{-j_{0}-1} R, 2^{-j_{0}+2} R\right)$. Also, we have

$$
\ell_{d^{c}}\left(\gamma_{1}\right)=\int_{0}^{\ell\left(\gamma_{1}\right)} \frac{d s}{d\left(c, \gamma_{1}(s)\right)^{2}} \approx \frac{\ell\left(\gamma_{1}\right)}{\left(2^{-j_{0}} R\right)^{2}} \leq C \frac{d\left(x_{0}, x_{1}\right)}{\left(2^{-j_{0}} R\right)^{2}} \lesssim d^{c}\left(x_{0}, x_{1}\right) .
$$

By induction, suppose $x_{i-1}$ has been chosen and take $x_{i} \in \gamma_{x_{i-1} y}$ (subcurve of $\gamma$ connecting $x_{i}$ and $\left.y\right)$ satisfying $\ell\left(\gamma_{x_{i-1} x_{i}}\right)=2^{-j_{i-1}} R /\left(2 C^{\prime}\right)$ for some $j_{i} \in \mathbf{N}$. If $\ell\left(\gamma_{x_{i-1} y}\right) \leq 2^{-j_{i-1}} R /\left(2 C^{\prime}\right)$, then just let $i=N$ and $x_{i}=y$. As the argument above, we can replace $\gamma_{x_{i-1} x_{i}}$ by $\gamma_{i}$ with the same endpoints, and we have $\ell_{d^{c}}\left(\gamma_{i}\right) \leq C d\left(x_{i-1}, x_{i}\right)$, for some $C$ depending only on the $\infty$-Poincare inequality of $(X, d, \mu)$ and the quasiconvexity constant of $\left(X^{c}, d^{c}\right)$.

In equations (4.5) and (4.6), we have shown that $\gamma \subset X \backslash B\left(c, 2^{-m} R\right)$ for some $m \geq k+2$. With the same argument, we can show that for every $1 \leq i \leq N$, $\gamma_{i} \subset X \backslash B\left(c, 2^{-m-1} R\right)$, so we can get $j_{i} \leq m+1$ for all $i$. Therefore, we can find 
an integer $N$ such that $x_{N}=y$, which means that this construction ends up after a finite number of steps.

To finish, take $\gamma^{\prime}$ as the concatenation of $\gamma_{1}, \gamma_{2}, \cdots, \gamma_{N}$, that has

$$
\ell_{d^{c}}\left(\gamma^{\prime}\right) \leq C \sum_{i=1}^{N} d^{c}\left(x_{i-1}, x_{i}\right) \leq C \ell_{d^{c}}(\gamma) \leq C d^{c}(x, y) .
$$

Therefore, we have shown that $\gamma^{\prime}$ is a quasiconvex curve connecting $x$ and $y$ with $\gamma^{\prime} \notin \Gamma_{N}^{+}$.

Example 4.8. Observe that we cannot remove the hypothesis of meridian-like quasiconvexity from Theorem 4.6 or Theorem 4.7. This example is considered in [2, Example 3.6.] as a metric space that fails to have a reverse scape property. Let $a_{i}=\frac{1}{(i+1) !}$ and $b_{i}=\frac{1}{i !}, i=2,3, \ldots$, and set

$$
X=[0,1] \times\{0\} \cup \bigcup_{i=1}^{\infty}\left\{a_{i}\right\} \times\left[0, b_{i}\right] \subset \mathbf{R}^{2},
$$

where $X$ is endowed with the length metric on $\mathbf{R}^{2}$ and the 1-dimensional Hausdorff measure $\mu$. Let $c=(0,0)$ be the point to be moved to $\infty$, and denote $x_{i}=\left(a_{i}, 0\right), y_{i}=$ $\left(a_{i}, b_{i}\right), a=(1,0)$. We can see that $\frac{\left|y_{i}-x_{i}\right|}{\left|x_{i}-c\right|}=\frac{(i+1) !}{i !}=i+1 \rightarrow \infty$, so $X$ is not meridianlike quasiconvex. We want to show that $(X, d, \mu)$ supports an $\infty$-Poincaré inequality, but $\left(X^{c}, d^{c}, \mu^{c}\right)$ does not. First, one can show that $(X, d, \mu)$ is Ahlfors 1-regular. Since $X$ is a length space, it is in particular quasiconvex. However, the flattened space $\left(X^{c}, d^{c}, \mu^{c}\right)$ is not quasiconvex. Let $i<j$. Then

$$
d\left(y_{i}, c\right)=\frac{1}{i !}+\frac{1}{(i+1) !}, \quad d\left(y_{j}, c\right)=\frac{1}{j !}+\frac{1}{(j+1) !},
$$

and

$$
d\left(y_{i}, y_{j}\right)=\frac{1}{i !}+\frac{1}{j !}+\frac{1}{(i+1) !}-\frac{1}{(j+1) !} \approx \frac{1}{i !} .
$$

Then we can get

$$
d^{c}\left(y_{i}, y_{j}\right)=\frac{d\left(y_{i}, y_{j}\right)}{d\left(y_{i}, c\right) d\left(y_{j}, c\right)} \approx \frac{\frac{1}{i !}}{\frac{1}{i !} \frac{1}{j !}}=j !
$$

On the other hand, since $(X, d, \mu)$ is a tree, every geodesic curve $\gamma$ connecting $y_{i}$ to $y_{j}$ is of the form $\left[x_{i}, y_{i}\right] \cup\left[x_{i}, x_{j}\right] \cup\left[x_{j}, y_{j}\right]$. We only need to estimate $\ell_{d^{c}}\left(\left[x_{j}, y_{j}\right]\right)$.

$$
\begin{aligned}
\ell_{d^{c}}\left(\left[x_{j}, y_{j}\right]\right) & =\int_{\left[x_{j}, y_{j}\right]} \frac{d w}{(d(w, c))^{2}}=\int_{0}^{\frac{1}{j !}} \frac{d t}{\left(\frac{1}{(j+1) !}+t\right)^{2}}=\frac{1}{\frac{1}{(j+1) !}-\frac{1}{\frac{1}{(j+1) !}+\frac{1}{j !}}} \\
& =(j+1) !-\frac{(j+1) !}{j+2} \approx(j+1) !
\end{aligned}
$$

Therefore, since $\frac{\ell_{d}^{c}(\gamma)}{d^{c}\left(y_{i}, y_{j}\right)} \approx \frac{(j+1) !}{j !} \approx j+1 \rightarrow \infty$ when $j \rightarrow \infty$, so $\left(X^{c}, d^{c}, \mu^{c}\right)$ is not quasiconvex and so cannot support an $\infty$-Poincaré inequality.

Acknowledgements. The authors would like to thank Prof. Shanmugalingam for proposing us the problem and for many fruitful discussions regarding this paper. We would also like to thank the anonymous referees for a very careful read of our paper and all the feedback received helping us to improve the final version of the manuscript. 


\section{References}

[1] Adamowicz, T., A. Buörn, J. Buörn, and N. Shanmugalingam: Prime ends for domains in metric spaces. - Adv. Math. 238, 2013, 459-505.

[2] Balogh, Z., and S. Buckley: Sphericalization and flattening. - Conform. Geom. Dyn. 9, 2005, 76-101.

[3] BJÖRn, A., and J. BJÖRn: Nonlinear potential theory on metric spaces. - EMS Tracts Math. 17, Eur. Math. Soc., Zürich, 2011.

[4] Buörn, J., and N. Shanmugalingam: Poincaré inequalities, uniform domains and extension properties for Newton-Sobolev functions in metric spaces. - J. Math. Anal. Appl. 332, 2007, 190-208.

[5] Bonk, M., J. Heinonen, and P. Koskela: Uniformizing Gromov hyperbolic spaces. Astérisque 270, 2001.

[6] Bonk, M., and B. Kleiner: Rigidity for quasi-Möbius group actions. - J. Differential Geom. 61, 2002, 81-106.

[7] Bonk, M., and O. Schramm: Embeddings of Gromov hyperbolic spaces. - GAFA 10, 2000, $266-306$.

[8] Buckley, S., D. Herron, and X. XiE: Metric inversions and quasihyperbolic geometry. Indiana Univ. Math. J. 57:2, 2008, 837-890.

[9] Cheeger, J.: Differentiability of Lipschitz functions on metric measure spaces. - Geom. Funct. Anal. 9, 1999, 428-517.

[10] Durand-Cartagena, E., and X. Li: Preservation of $p$-Poincaré inequality for large $p$ under sphericalization and flattening. - Preprint, http://cvgmt.sns.it/paper $/ 2571 /$.

[11] Durand-Cartagena, E., N. Shanmugalingam, and J. Jaramillo: Geometric characterizations of $p$-Poincaré inequalities in the metric setting. - Publ. Mat. 60, 2016, 81-111.

[12] Durand-Cartagena, E., N. Shanmugalingam, and A. Williams: p-Poincaré inequality vs. $\infty$-Poincaré inequality; some counter-examples. - Math. Z. 271, 2012, 447-467.

[13] Estep, E.: Prime end boundaries of domains in metric spaces and the Dirichlet problem. PhD Thesis, Univ. of Cincinnati.

[14] Estep, D., and N. Shanmugalingam: Geometry of prime end boundary and the Dirichlet problem for bounded domains in metric measure spaces. - Potential Anal. 42:2, 2015, 335-363.

[15] HajŁasz, P., and P. Koskela: Sobolev met Poincaré. - Mem. Amer. Math. Soc. 145:688, 2000.

[16] Heinonen, J.: Lectures on analysis on metric spaces. - Springer, New York, 2001.

[17] Heinonen, J., and P. Koskela: Quasiconformal maps in metric spaces with controlled geometry. - Acta Math. 181, 1998, 1-61.

[18] Heinonen, J., P. Koskela, N. Shanmugaligam, and J. Tyson: Sobolev spaces on metric measure spaces: an approach based on upper gradients. - New Math. Monogr., Cambridge Univ. Press, 2015.

[19] Herron, D.: Uniform metric spaces, annular quasiconvexity and pointed tangent spaces. Math. Scand. 108, 2011, 115-145.

[20] Herron, D., N. Shanmugalingam, and X. XIE: Uniformity from Gromov hyperbolicity. Illinois J. Math. 52:4, 2008, 1065-1109.

[21] Korte, R.: Geometric implications of the Poincaré inequality. - Results Math. 50:1-2, 2007, $93-107$.

[22] Li, X., and N. Shanmugalingam: Preservation of bounded geometry under sphericalization and flattening. - Indiana Univ. Math. J. 64:5, 2015, 1303-1341.

[23] Munkres, J. R.: Topology. 2nd edition. - Prentice Hall, 2000.

[24] Shanmugalingam, N.: Newtonian spaces: an extension of Sobolev spaces to metric measure spaces. - Rev. Mat. Iberoamericana 16:2, 2000, 243-279. 